\title{
FURTHER RESULTS ON ORDER TYPES AND DECOMPOSITIONS OF SETS
}

\author{
BY \\ SEYMOUR GINSBURG
}

In this paper the study of problems $\mathrm{P}$ and $\mathrm{Q}[3 ; 4]$ is continued. The reader is referred to the aforementioned two references for all unfamiliar terms and symbols.

The first three sections deal with the decomposition of a linear set into a family of pairwise disjoint sets, the order types of the sets being required to satisfy some specified conditions. $\$ 1$ is concerned with the decomposition of an arbitrary linear set, of power $2^{\aleph_{0}}$, into a family of pairwise disjoint sets, the order types of the sets being pairwise incomparable. $\$ 2$ is concerned with the decomposition of certain linear sets into families of pairwise disjoint, similar sets. $\S 3$ is concerned with the decomposition of an arbitrary linear set into families, of power $\boldsymbol{\aleph}_{0}$ and $2^{\aleph_{0}}$, of pairwise disjoint sets, each set having property $\mathrm{A}$.

Let $\left\{\alpha_{\xi}\right\}, \xi<\theta$, be a sequence of order types, of power $2^{N_{0}}$ each, such that $\alpha_{\xi}<\lambda$ for each $\xi$. In $\S 4$ it is shown that

(1) problem $\mathrm{P}$, as applied to $\sigma=\alpha_{\xi}$ and $\mu=\lambda$, admits of a solution $\tau_{\xi}$ such that the $\tau_{\xi}$ are pairwise incomparable order types (Theorem 4.1); and

(2) problem $\mathrm{P}$, as applied to each $\sigma=0$ and $\mu=\alpha_{\xi}$, admits of a solution $\tau_{\xi}$ such that the $\tau_{\xi}$ are pairwise incomparable order types (Theorem 4.3).

1. Decompositions into incomparable order types. In this section the decomposition of a linear set into a finite number and into a denumerably infinite number of pairwise disjoint sets $A_{i}$, where the order types of the $A_{i}$ are pairwise incomparable, is studied. The decomposition of a linear set into $2^{\aleph_{0}}$ pairwise disjoint sets $A_{i}$, where the order types of the $A_{i}$ are pairwise incomparable, is treated in $\$ 3$ (Theorem 3.4).

Definition. A linear set $E$, of power $2^{N_{0}}$, will be said to have property $\mathrm{C}$ if each element of $E$ is a $c$-condensation point of $E$.

For any linear set $E$, by $K(E)$ is meant the set of similarity transformations of $E$ into $R$. By $K^{*}(E)$ is meant the set $K(E)-\{I\}$, where $I$ is the identity transformation of $E$.

For any similarity transformation $f$ of $A$ into $B$, by $f^{*}$ is meant the inverse of $f$.

Lemma 1.1. Let $E$ be a linear set which has property C. For each ordinal number $\alpha$, where $2 \leqq \alpha \leqq \omega, E$ is the union of a family $\left\{A_{i} \mid i<\alpha\right\}$ of pairwise disjoint, exact sets, each of which has property $\mathrm{C}$ and is a dense subset of E. Fur-

Presented to the Society, April 24, 1953; received by the editors February 16, 1953. 
thermore, if $f$ is any element of $K\left(A_{i}\right)$ for which the power of the set $E \cap f\left(A_{i}\right)$ is $2^{\aleph_{0}}$, then there are $2^{\aleph_{0}}$ elements $x$ in $A_{i}$ such that $f(x)$ is also in $A_{i}$.

Proof. Since $E$ has property C, it follows that for each element $f$ in $K^{*}(E)$, the power of the set, $\{x \mid f(x) \neq x, x \in E\}$, is $2^{N_{0}}$. Well order the elements of $E$ and $K^{*}(E)$ into the two sequences, $\left\{x_{\xi}\right\}, \xi<\theta$, and $\left\{f_{\xi}\right\}, \xi<\theta$, where each element in $K^{*}(E)$ appears $2^{\aleph_{0}}$ times in the latter sequence. If $\alpha=\omega$, let $\beta=\alpha$. If $\alpha$ is an integer let $\beta=\alpha-1$. Suppose that for each ordinal number $\xi$, where $\xi<\mu<\theta$, the elements $p_{\xi}^{i, m}$, where $i=1,2, \cdots, 10$, and $m<\beta$, have been defined. Let

$X_{\mu}=\left\{x \mid f_{\mu}(x) \neq x, x \in E\right\} \quad$ and $\quad Y_{\mu}=\left\{p_{\xi}^{i, m} \mid \xi<\mu, m<\beta, 1 \leqq i \leqq 10\right\}$.

Denote by $\left\{p_{\mu}^{1, m} \mid m<\beta\right\}$ and by $\left\{p_{\mu}^{2, m} \mid m<\beta\right\}$ the first $\beta 2$ elements in the set $E-Y_{\mu}$. Let

$$
A_{\mu}=Y_{\mu} \cup\left\{p_{\mu}^{i, m} \mid i \leqq 2, m<\beta\right\} \quad \text { and } \quad B_{\mu}=X_{\mu}-\left[A_{\mu} \cup f_{\mu}^{*}\left(A_{\mu}\right)\right] .
$$

Let $p_{\mu}^{3,0}$ be the first element in $B_{\mu}$ and $p_{\mu}^{4,0}=f_{\mu}\left(p_{\mu}^{3,0}\right)$. Since the power of $X_{\mu}$ is $2^{\aleph_{0}}$ and the power of $A_{\mu}$ is $\left\langle 2^{\aleph_{0}}\right.$, the two distinct elements $p_{\mu}^{3,0}$ and $p_{\mu}^{4,0}$ certainly exist. In general, for $m<\beta$ let $p_{\mu}^{3, m}$ be the first element in the set $B_{\mu}-\left[G_{\mu}^{m} \cup f^{*}\left(G_{\mu}^{m}\right)\right]$, where

$$
G_{\mu}^{m}=\left\{p_{\mu}^{3, i} \mid i<m\right\} \cup\left\{p_{\mu}^{4, i} \mid i<m\right\} .
$$

Let $p_{\mu}^{4, m}=f_{\mu}\left(p_{\mu}^{3, m}\right)$. Let

$$
C_{\mu}=Y_{\mu} \cup\left\{p_{\mu}^{i, m} \mid i \leqq 4, m<\beta\right\} \quad \text { and } D_{\mu}=X_{\mu}-\left[C_{\mu} \cup f_{\mu}^{*}\left(C_{\mu}\right)\right] .
$$

Let $p_{\mu}^{6,0}$ be the first element in $D_{\mu}$ and $p_{\mu}^{5,0}=f_{\mu}\left(p_{\mu}^{6,0}\right)$. Let $p_{\mu}^{5, \imath}$ and $p_{\mu}^{6, t}, 1 \leqq i<\beta$, represent no elements. Denote by $Z_{\mu}$ the set $Z_{\mu}=\left\{x \mid x \in E, f_{\mu}(x) \in E\right\}$. Suppose that the power of $Z_{\mu}$ is $2^{\aleph_{0}}$. Let

$$
E_{\mu}=Y_{\mu} \cup\left\{p_{\mu}^{i, m} \mid i \leqq 6, m<\beta\right\} \quad \text { and } \quad F_{\mu}=Z_{\mu}-\left[E_{\mu} \cup f_{\mu}^{*}\left(E_{\mu}\right)\right] .
$$

Let $p_{\mu}^{7,0}$ be the first element in $F_{\mu}$ and $p_{\mu}^{9,0}=f_{\mu}\left(p_{\mu}^{7,0}\right)$. In general, for $m<\beta$ let $p_{\mu}^{7, m}$ be the first element in the set $F_{\mu}-\left[H_{\mu}^{m} \cup f_{\mu}^{*}\left(H_{\mu}^{m}\right)\right]$, where

$$
H_{\mu}^{m}=\left\{p_{\mu}^{7, i} \mid i<m\right\} \cup\left\{p_{\mu}^{9, i} \mid i<m\right\},
$$

and $p_{\mu}^{9, m}=f_{\mu}\left(p_{\mu}^{7, m}\right)$. Let

$$
M_{\mu}=\left\{p_{\mu}^{7, i} \mid i<\beta\right\} \cup\left\{p_{\mu}^{9, i} \mid i<\beta\right\} .
$$

Denote by $p_{\mu}^{8,0}$ the first element in the set $F_{\mu}-\left[M_{\mu} \cup f_{\mu}^{*}\left(M_{\mu}\right)\right]$, and by $p_{\mu}^{10,0}$, the element $f_{\mu}\left(p_{\mu}^{8,0}\right)$. For $1 \leqq i<\beta$ let $p_{\mu}^{8,4}$ and $p_{\mu}^{10, i}$ represent no elements. If the power of $Z_{\mu}$ is $<2^{\aleph_{0}}$, let $p_{\mu}^{i, m}$, where $7 \leqq i \leqq 10$ and $m<\beta$, represent no elements. For each $i<\beta$, let 


$$
\begin{aligned}
& A_{i}=E \cap\left\{p_{\mu}^{2 n+1, i} \mid n \leqq 4, \mu<\theta\right\} \text { and } \\
& A_{\omega}=E \cap\left\{p_{\mu}^{2 n+2, i} \mid i<\beta, n \leqq 4, \mu<\theta\right\} .
\end{aligned}
$$

The sets $A_{\gamma}$ are evidently pairwise disjoint. Let $J$ be any open interval which contains a point of $E$. There exists an elements $f_{\mu}$ of $K^{*}(E)$ which is the identity on $E-J$ and for which $f_{\mu}(x) \neq x$ when $x$ is in $J \cap E$. Consequently the elements $p_{\mu}^{1, m}$ and $p_{\mu}^{2, m}$ are in the set $J \cap E$. This shows that for each $\gamma, A_{\gamma}$ is a dense subset of $E$. As each element in $K^{*}(E)$ occurs $2^{\aleph_{0}}$ times in the sequence $\left\{f_{\xi}\right\}, \xi<\theta$, each point of $A_{\gamma}$ is a $c$-condensation point of $A_{\gamma}$. From the selection of the elements $p_{\mu}^{1, m}$ and $p_{\mu}^{2, m}$, it follows that the set union of the sets $A_{\gamma}$ is $E$. Since $A_{\gamma}$ is a dense subset of $E$ and $E$ has property $\mathrm{C}$, each element $f$ of $K^{*}\left(A_{\gamma}\right)$ can be extended to be an element of $K^{*}(E)$. From this and the manner in which the elements $p_{\mu}^{i, m}, 3 \leqq i \leqq 6$, were chosen, it follows that $f\left(A_{\gamma}\right)$ is not a subset of $A_{\gamma}$. Therefore $A_{\gamma}$ is exact. The manner of selection of the elements $p_{\mu}^{i, m}, i \geqq 7$, guarantees that for each element $f$ in $K\left(A_{\gamma}\right)$ such that the power of the set $E \cap f\left(A_{\gamma}\right)$ is $2^{\aleph_{0}}$, there are $2^{\aleph_{0}}$ elements $x$ in $A_{\gamma}$ such that $f(x)$ is also in $A_{\gamma}$. Q.E.D.

Suppose that $f$ is a similarity transformation of one of the sets $A_{i}$ into one of the sets $A_{j}$, where $i \neq j$. Since $f\left(A_{i}\right)$ is a subset of $A_{j}$, the power of the set $f\left(A_{i}\right) \cap E$ is $2^{\aleph_{0}}$. Therefore there are $2^{\aleph_{0}}$ elements $x$ in $A_{i}$ such that $f(x)$ is also in $A_{i}$, thus not in $A_{j}$. Consequently $f \operatorname{does}$ not map $A_{i}$ into $A_{j}$, i.e.,

Theorem 1.1. Let $E$ and $\alpha$ be the same as in Lemma 1.1. Then $E$ is the union of a family $\left\{A_{i} \mid i<\alpha\right\}$ of pairwise disjoint, exact sets, of power $2^{\aleph_{0}}$ each, such that the $\bar{A}_{i}$ are pairwise incomparable order types.

CoROllaRY. For each ordinal number $\alpha$, where $2 \leqq \alpha \leqq \omega, R$ is the union of $a$ family $\left\{A_{i} \mid i<\alpha\right\}$ of pairwise disjoint sets, which have the following properties:

(a) For each $i, A_{i}$ is an exact, dense subset of $R$.

(b) Each set $A_{i}$ has property $\mathrm{C}$.

(c) The $\bar{A}_{i}$ are pairwise incomparable order types.

If, in Theorem 1.1, the assumption that $E$ has property $\mathrm{C}$ is removed, then some of the conclusions are no longer valid. In particular, it is no longer true that each set $A_{i}$ can be made exact. To see this consider a set $E$ which is defined as an ordered sum $E=B+C+D\left({ }^{1}\right)$, where $C$ is denumerably infinite. Now let $E$ be the union of $n+1$ disjoint sets $E_{i}$, where $n$ is some non-negative integer. For at least one of the sets, say $E_{j}$, the set $E_{j} \cap C=G$ is denumerably

(1) Let $\left\{E_{a} \mid a \in A\right\}$ be a family of (not necessarily nonempty) pairwise disjoint simply ordered sets, where $A$ is a nonempty simply ordered set. By the ordered sum $\sum_{a \in A} E_{a}$ is meant the set union of the $E_{a}, M=\bigcup_{a \in A} E_{a}$, the elements being ordered as follows: If $u<v$ in $E_{a}$, where $u$ and $v$ are two elements in $E_{a}$, then $u<v$ in $M$. If $u$ is in $E_{a}$ and $v$ is in $E_{b}$, where $a<b$, then $u<v$ in $M$. 
infinite. Let $E_{j}$ be the ordered sum $F+G+H$. In [2, pp. 322-323] it was shown that no denumerably infinite set is exact. From the remark following Corollary 1 of Theorem 2 in [3], the set $E_{j}$ is not exact. Thus we have demonstrated the following result:

Theorem 1.2. A necessary condition that $E$ be the union of a finite number of disjoint exact sets is that $E$ not be the ordered sum $B+C+D$, where $C$ is denumerably infinite.

As a modification of Theorem 1.1 we have

Theorem 1.3. Let $A$ be any linear set of power $2^{\aleph_{0}}$. For each ordinal number $\alpha$, where $2 \leqq \alpha \leqq \omega, A$ is the union of a family $\left\{E_{i} \mid i<\alpha\right\}$ of pairwise disjoint sets, of power $2^{\aleph_{0}}$ each, such that the $\bar{E}_{i}$ are pairwise incomparable order types.

Proof. Let $E$ be the set of $c$-condensation points of $A$ which belong to $A$. As is well known, the power of the set $A-E$ is $\left\langle 2{ }^{N_{0}}\right.$. The set $E$ satisfies the assumptions of Lemma 1.1. Let $\left\{A_{i} \mid i<\alpha\right\}$ be the family of sets obtained from the conclusion of the lemma. Let $E_{0}=A_{0} \cup(A-E)$, and for $i \geqq 1$, let $E_{i}=A_{i}$. Suppose that $f\left(E_{i}\right)$ is a subset of $E_{j}$, where $i \neq j$, for some element $f$ in $K\left(E_{i}\right)$. Then the power of the set $E \cap f\left(A_{i}\right)$ is $2^{\aleph_{0}}$. Since there are $2^{\aleph_{0}}$ elements $x$ in $A_{i}$ for which $f(x)$ is also in $A_{i}$, and since the $E_{i}$ are pairwise disjoint, it follows that $f\left(A_{i}\right)$, thus $f\left(E_{i}\right)$, is not a subset of $E_{j}$. Thus the function $f$ cannot exist. Therefore the order types of the $E_{i}$ are pairwise incomparable.

COROLlARY. In Lemma 1.1 let property $\mathrm{C}$ be replaced by the following property: Except for a finite number of points, each element of $E$ is a c-condensation point of $E$. Then for each ordinal number $\alpha$, where $2 \leqq \alpha \leqq \omega, E$ is the union of a family $\left\{A_{i} \mid i<\alpha\right\}$ of pairwise disjoint, exact sets, of power $2^{\aleph_{0}}$ each, such that if $f$ is any element of $K\left(A_{i}\right)$ for which the power of the set $E \cap f\left(A_{i}\right)$ is $2 \mathbf{N}_{0}$, then there are $2^{\aleph_{0}}$ elements $x$ in $A_{i}$ such that $f(x)$ is also in $A_{i}$.

REMARK. There is no difficulty in extending Lemma 1.1 and Theorems 1.1 and 1.3 to hold in the case where $2 \leqq \alpha<\theta$.

THEOREM 1.4. If $2^{\boldsymbol{N}_{0}}=\boldsymbol{\aleph}_{1}$, then each linear set $A$, of power $2^{\boldsymbol{\aleph}_{0}}$, is the union of a family $\left\{E_{n} \mid n<\omega\right\}$ of pairwise disjoint, exact sets, of power $2^{N_{0}}$ each, such that the order types of the $E_{n}$ are pairwise incomparable.

Proof. Let $E$ be the set of $c$-condensation points of $A$ which belong to $A$. Since $2^{\aleph_{0}}=\aleph_{1}$ and the power of the set $A-E$ is $<2 \aleph_{0}, A-E$ is denumerable. Let $A-E=\left\{x_{i} \mid i<\mu \leqq \omega\right\}$. Let $\left\{A_{i} \mid i<\omega\right\}$ be the family of sets obtained from the conclusion of Lemma 1.1. For $i<\mu$ let $E_{i}=A_{i} \cup\left\{x_{i}\right\}$, and for $i \geqq \mu$ let $E_{i}=A_{i}$. Since $A_{i}$ is exact, the set $A_{i} \cup\left\{x_{i}\right\}$ is exact. The pairwise incomparability of the order types of the $E_{i}$ follows from an argument similar to that given in Theorem 1.3. 
REMARK. The conclusion of Theorem 1.4 will be strengthened later to require that each set $E_{n}$ have property $\mathrm{A}$ (Theorem 3.2).

We conclude this section with denumerable sets.

LEMмA 1.2. Let $S$ be a set such that $\bar{S} \equiv \eta$. If $S$ is the union of a finite family $\left\{A_{i} \mid i<n\right\}$ of pairwise disjoint sets, then for at least one integer $i, \bar{A}_{i} \equiv \eta$.

Proof. For each denumerable ordinal number $\xi, \xi \leqq \omega^{\xi}\left[5\right.$, p. 107]. Now $\omega^{\xi}$ is denumerable. For assume that $\omega^{v}$ is denumerable for each $v<\xi$. If $\xi=\gamma+1$, then $\omega^{\xi}=\omega^{\gamma} \omega$. If $\xi$ is a limit number, then, by definition, $\omega^{\xi}=\lim _{v<\xi} \omega^{v}$. Being either the product of two, or the limit of a denumerable number of denumerable ordinal numbers, $\omega^{\xi}$ is denumerable. Let $B_{\xi}$ be a subset of $S$ of order type $\omega^{\xi}$. Since $S$ is the union of the finite number of sets $A_{i}$, one of the sets, say $A_{i(\xi)}$, contains a subset of $B_{\xi}$ of order type $\omega^{\xi}$. Therefore one of the sets, say $A_{j}$, has the property that for each denumerable ordinal number $\xi, A_{j}$ contains a subset of order type $v \geqq \xi$. This implies that for each denumerable ordinal number $\xi, A_{j}$ contains a subset of order type $\xi$. It is known that this is sufficient for $\bar{A}_{j} \equiv \eta$ to hold [6].

A consequence of the lemma is

THEOREM 1.5. If $\bar{S} \equiv \eta$, then $S$ is not the union of a finite family $\left\{A_{i} \mid i<n\right\}$ of pairwise disjoint sets such that the $\bar{A}_{i}$ are pairwise incomparable order types.

2. Decompositions into similar sets. Due to the existence of sets which have property $\mathrm{A}$, it is impossible to obtain results on decompositions into sets whose order types are equal as general as the results on decompositions into sets whose order types are pairwise incomparable.

Several decompositions of $R$ into families of $\aleph_{0}$ and $2^{\aleph_{0}}$ pairwise disjoint, similar sets, of power $2^{\aleph_{0}}$ each, are already in the literature, e.g. $[7 ; 12 ; 15]$. Whether or not the sets obtained there are exact is an open problem. By Corollary 3.2 of [4], $R$ cannot be the union of two disjoint, similar, exact sets. Whether or not $R$ is the union of $n$ disjoint, similar, exact sets, $n$ being an integer $>2$, is unknown. In this section we shall show that $R$ is the union of families of $\boldsymbol{N}_{0}$ and $2^{\boldsymbol{N}_{0}}$ pairwise disjoint, similar, exact sets, of power $2^{\boldsymbol{N}_{0}}$ each (Theorem 2.1 and Corollary 2 of Theorem 2.2).

LEMмa 2.1. If $\bar{E}=\bar{D}_{0}\left(\omega^{*}+\omega\right)$, where $D_{0}$ is the union of two disjoint, exact sets (of power $2 \aleph_{0}$ each), $B$ and $C$, then $E$ is the union of a family of $\aleph_{0}$ pairwise disjoint, similar, exact sets (of power $2^{\aleph_{0}}$ each).

Proof. Since $\bar{E}=\bar{D}_{0}\left(\omega^{*}+\omega\right), E$ is the ordered sum of the sets

$$
E=\cdots+D_{-i}+\cdots+D_{0}+D_{1}+\cdots+D_{j}+\cdots \text {, }
$$

each set $D_{i}, i=0, \pm 1, \pm 2, \cdots$, being similar to $D_{0}$. Let $f_{0}$ be the identity transformation of $D_{0}$ and $f_{i}$ a similarity transformation of $D_{0}$ onto $D_{i}$. It follows from Theorem 2 of [3] that the family of sets 


$$
\left\{A_{i}=f_{i}(B) \cup f_{i+1}(C) \mid i= \pm n, n<\omega\right\}
$$

satisfy the conclusion of the lemma.

Since $R=\{(i, i+1] \mid i= \pm n, n<\omega\}$, we obtain from Lemma 2.1 and the corollary to Theorem 1.3

THeOREM 2.1. $R$ is the union of a family $\left\{E_{n} \mid n<\omega\right\}$ of pairwise disjoint, similar, exact sets, of power $2^{N_{0}}$ each.

Open Questions. (1) Can the family of sets in Theorem 2.1 be chosen so that each set has property $A$ ?

(2) Let $E$ be any linear set of power $2^{N_{0}}$. Is $R$ the union of a family $\left\{E_{n} \mid n<\omega\right\}$ of pairwise disjoint sets, each set being similar to $E$ ?

Remark. It is not true that every linear set $E$, such that $\bar{E} \equiv \lambda$, is the union of a family $\left\{A_{n} \mid n<\omega\right\}$ of pairwise disjoint, similar, exact sets. Consider the following example. Let $B$ be a dense subset of $(0,1)$, which has properties $A$ and $C$. The existence of such a set is guaranteed by Lemma 1 of [3] and Theorem 5 of [3]. The set $E$ is defined to be

$$
E=B \cup\{2-1 / n \mid 1 \leqq n<\omega\} \cup(3,4) \text {. }
$$

Evidently $\bar{E} \equiv \lambda$. Suppose that $E$ is the union of a family $\left\{A_{n} \mid n<\omega\right\}$ of pairwise disjoint, similar sets. Since $B$ has property A, no two of the sets, $A_{i}$ and $A_{j}$, each contain $2^{\aleph_{0}}$ elements of $B$. Let $G_{i}=B \cap A_{i}$. Then $2^{\aleph_{0}}$ $=\sum_{n<\omega}$ top $\left(G_{n}\right)$, top $\left(G_{n}\right)$ designating the power of $G_{n}$. By König's Theorem $\left[5\right.$, p. 45], the power of one of the sets, say $G_{0}$, is $2^{\aleph_{0}}$. Since $B$ has property A, at most one of the sets $G_{i}$ is of power $2^{\mathbb{N}_{0}}$. Thus top $\left(G_{i}\right)<2 \mathbb{N}_{0}$ for $i>0$. Thus the power of the set $U_{i>0} G_{i}$ is $\left\langle 2^{N_{0}}\right.$. Now the set $B$ has the property that if $a$ and $b$ are any two elements in $B$, then the power of the set $\{x \mid a<x<b, x \in B\}$ is $2^{N_{0}}$. As $G_{0}=B-\bigcup_{i>0} G_{i}$, it follows that $G_{0}$ has the same property, i.e., if $a$ and $b$ are any two elements in $G_{0}$, then the power of the set $\{x \mid a<x<b$, $\left.x \in G_{0}\right\}$ is $2^{\aleph_{0}}$. Now $\bar{A}_{0}=\bar{A}_{i}$. Thus, for each $i>0$, if $G_{i}$ had at least two elements, it would have to have $2^{\aleph_{0}}$ elements, a contradiction. Hence, for each $i>0, G_{i}$ has at most one element, say $a_{i}$. But $G_{0}$ has no first element. Therefore $G_{i}$ must be empty, i.e., $G_{0}=B$. Since $A_{0}$ has no first element, $A_{i}$ has no first element. Therefore $G_{0}$ contains the set $\{2-1 / n \mid 1 \leqq n<\omega\}$. By Theorem 1.2 , the set $A_{0}$ cannot be exact.

We now prove a general theorem on decompositions of $R$.

TheOREM 2.2. Let $\left\{\alpha_{\xi}\right\}, \xi<\theta$, be a sequence of linear order types, where $\alpha_{\xi} \neq 0$. Then $R$ is the union of a family $\left\{E_{\xi} \mid \xi<\theta\right\}$ of pairwise disjoint sets, such that $\bar{E}_{\xi}=\alpha \xi$.

Proof. Let $C$ be the Cantor set, i.e., all numbers of the form $x=\sum_{i=1}^{\infty} a_{i} / 3^{i}$, where $a_{i}=0$ or 2. For each sequence of 0 's and 2's, say $t_{1} t_{3} t_{5} \cdots$, the set

$$
C\left(t_{1} t_{3} t_{5} \cdots\right)=\left\{x \mid x \in C, a_{i}=t_{i} \text { for all odd } i\right\}
$$


is a perfect set. The family of all such sets is then a family of $2^{\aleph_{0}}$ pairwise disjoint perfect sets. Under a mapping of the form $a x+b, a \neq 0$, a perfect set is mapped into a perfect set. Thus each interval of $R$ contains a family of $2^{\mathbf{N}_{0}}$ pairwise disjoint perfect sets. For each integer $n$, which is positive, negative, or 0 , let $\left\{P_{\xi}^{n} \mid \xi<\theta\right\}$ and $\left\{Q_{\xi}^{n} \mid \xi<\theta\right\}$ be two families of pairwise disjoint, perfect sets such that $Q_{\xi}^{n}$ and $P_{\xi}^{n}$ are subsets of the open intervals $(n, n+1 / 2)$ and $(n+1 / 2, n+1)$ respectively. Furthermore, for each $n$

$(*)$ let the power of the two sets,

$$
(n, n+1 / 2)-\bigcup_{\xi<\theta} Q_{\xi}^{n} \text { and }(n+1 / 2, n+1)-\bigcup_{\xi<\theta} P_{\xi}^{n}
$$

be $2^{\aleph_{0}}$ each.

Denote by $Z=\left\{Y_{v} \mid v<\theta\right\}$ the family of sets

$$
Z=\left\{\left(P_{\xi}^{i-1} \cup Q_{\xi}^{i+1}\right) \mid i= \pm n, n<\omega, \xi<\theta\right\} .
$$

For each $v<\theta$ define $A_{v}$ and $B_{v}$ to be the $P$-set and $Q$-set respectively of $Y_{v}$. For each $v<\theta$ there exist two order types $\sigma_{v}$ and $\tau_{v}$ (possibly 0 ) such that $\alpha_{v}=\sigma_{v}+1+\tau_{v}$. Let $C_{v}$ be a subset of $A_{v}$ having order type $\sigma_{v}$, and $D_{v}$ a subset of $B_{v}$ having order type $\tau_{v}$. The sets $C_{v}$ and $D_{v}$ exist since, $P_{\xi}^{n}$ being a perfect set, $\overline{P_{\xi}^{n}} \equiv \lambda$. Let $H$ be the set $R-\bigcup_{v<\theta}\left[C_{v} \cup D_{v}\right]$. By $\left(^{*}\right)$ the power of $H$ is $2^{\aleph_{0}}$. Well order the elements of $H$ into the sequence $\left\{x_{\xi}\right\}, \xi<\theta$. Assume that the set $E_{v}=C_{v} \cup D_{v} \cup\left\{y_{v}\right\}$ has been defined for each ordinal number $v$, where $v<\mu<\theta$. Denote by $y_{\mu}$ the first element in the set $H-\left\{y_{v} \mid v<\mu\right\}$ such that $u<y_{\mu}<v$ for each element $u$ in $A_{\mu}$ and $v$ in $B_{\mu}$. From $\left({ }^{*}\right)$ and the fact that an interval $(n, n+1)$ lies between $A_{\mu}$ and $B_{\mu}$ the element $y_{\mu}$ exists. Denote by $E_{\mu}$ the set $C_{\mu_{v}} \cup D_{\mu} \cup\left\{y_{\mu}\right\}$. Obviously the $E_{\mu}$ are pairwise disjoint sets such that $\bar{E}_{\mu}=\alpha_{\mu}$. There is no difficulty in seeing that each element $x_{\xi}$ is one of the $y_{v}$. In fact, consider the element $x_{\xi}$ of $H$. Suppose that $x_{\xi}$ is in the closed interval $[n, n+1]$. Let $Y_{v}$ be the $\xi$ th set in $Z$ whose $P$-set and $Q$-set are separated by $[n, n+1]$. From the selection of the points $y_{\mu}, x_{\xi}$ must be in the set $\left\{y_{\mu} \mid \mu \leqq \xi\right\}$. Consequently the union of the $E_{\mu}$ is $R$. Q.E.D.

COROLlaRy 1. For each linear order type $\alpha, \alpha \neq 0, R$ is the union of a family $\left\{E_{\xi} \mid \xi<\theta\right\}$ of pairwise disjoint sets, each of order type $\alpha$.

Corollary 2. $R$ is the union of a family, $\left\{E_{\xi} \mid \xi<\theta\right\}$, of pairwise disjoint, similar, exact sets, each having property A.

The same method of proof may be used to show

THEOREM 2.3. Let $\left\{\bar{G}_{\xi}\right\}, \xi<\theta$, be a sequence of linear order types such that for $2^{N_{0}}$ ordinal numbers $\xi, G_{\xi}$ has both a first and a last element. Then each set $E$ for which $\bar{E} \equiv \lambda$ is the union of a family $\left\{E_{\xi} \mid \xi<\theta\right\}$ of pairwise disjoint sets such that $\bar{G}_{\xi}=\bar{E}_{\xi}$ for each $\xi<\theta$. 
The problem of whether or not, for $2^{N_{0}}$ ordinal numbers $\xi, G_{\xi}$ must have both a first and a last element in order that the conclusion of Theorem 2.3 hold for every set $E$ for which $\bar{E} \equiv \lambda$ remains unanswered. In the special case that $\bar{G}_{\xi}=\bar{G}_{v}$ for $\xi \neq v$ the answer is obviously in the affirmative (consider when $E$ is the closed interval $[a, b])$, i.e.,

THEOREM 2.4. Let $B$ be a linear set. A necessary and sufficient condition that each set $E$, where $\bar{E} \equiv \lambda$, be the union of a family $\left\{E_{\xi} \mid \xi<\theta\right\}$ of pairwise disjoint sets, each of order type $\bar{B}$, is that $B$ have both a first and a last element.

We now consider a general result on the decomposition of a linear set into a family of $2{ }^{\aleph_{0}}$ pairwise disjoint, similar, exact sets, each set having property A.

THEOREM 2.5. Let $E$ be a linear set which contains a family $E=\left\{E_{\xi} \mid \xi<\theta\right\}$ of pairwise disjoint, similar subsets, of power $2^{\aleph_{0}}$ each. Then $E$ is the union of a family $T=\left\{A_{\xi} \mid \xi<\theta\right\}$ of pairwise disjoint, similar, exact sets, each of which has property A. Furthermore, $\bar{A}_{\xi}<\bar{E}_{\xi}$.

In order to prove the theorem we first prove two lemmas.

LEMMA 2.2. Let $F=\left\{E_{\xi} \mid \xi<\theta\right\}$ be a family of pairwise disjoint, similar, linear sets, of power $2^{N_{0}}$ each. Then there exists a family $G=\left\{B_{\xi} \mid \xi<\theta\right\}$ of similar subsets, of power $2{ }^{N_{0}}$ each, and a closed interval $[p, q]$ of $R$, with the following properties:

(a) Each element of $G$ is a subset of $[p, q]$.

(b) Each element of $G$ is a subset of an element of $F$, and each element of $F$ contains, at most, one element of $G$ (thus the $B_{\xi}$ are pairwise disjoint).

(c) For each $\xi<\theta$, if $u$ and $v$, where $u<v$, are any two elements of $B_{\xi}$, then the power of the set $\left\{x \mid u<x<v, x \in B_{\xi}\right\}$ is $2^{\aleph_{0}}$.

(d) There are $2^{\aleph_{0}}$ elements $x$ in the set $U_{\xi<\theta} E_{\xi}$ such that $x<p$, and there are $2^{\mathrm{N}_{0}}$ elements $x$ in the set $\mathrm{U}_{\xi<\theta} E_{\xi}$ such that $x>q$.

Proof. For each $\xi<\theta$ let $f_{\xi}$ be a similarity transformation of $E_{0}$ onto $E_{\xi}$. Denote by $C$ the set of $c$-condensation points of $E_{0}$ which are in $E_{0}$. Let $I_{0}$ be an interval of $C$ which has a first element $a$ and a last element $b, a \neq b$. Let $p$ be a $c$-condensation point of the set $H=\left\{f_{\xi}(a) \mid \xi<\theta\right\}$ which is in $H$, and let $K=\left\{v \mid f_{v}(a) \geqq p\right\}$. Let $q$ be a $c$-condensation point of the set $J$ $=\left\{f_{v}(b) \mid v \in K\right\}$ which is in $J$, and let $L=\left\{v \mid f_{v}(b) \leqq q, v \in K\right\}$. The power of $L$ is $2^{N_{0}}$. Denote by $G=\left\{B_{\xi} \mid \xi<\theta\right\}$ the family of sets $\left\{f_{v}(I) \mid v \in L\right\}$. The family $G$ satisfies the conclusion of the lemma.

LEMMA 2.3. If $G=\left\{B_{\xi} \mid \xi<\theta\right\}$ is a family of sets which satisfy the conclusion of Lemma 2.2, then there exists a family $M=\left\{D_{\xi} \mid \xi<\theta\right\}$ of similar sets, of power $2^{N_{0}}$ each, with the following properties:

(1) Each element of $M$ is a subset of an element of $G$ (thus the $D_{\xi}$ are pairwise disjoint). 
(2) $M$ is the union of two disjoint families, $Y=\left\{P_{\xi} \mid \xi<\theta\right\}$ and $Z$ $=\left\{Q_{v} \mid v<\theta\right\}$, such that $x<y$ for all elements $x$ in $P_{\xi}$ and $y$ in $Q_{v}$.

Proof. For each $\xi<\theta$ let $g_{\xi}$ be a similarity transformation of $B_{0}$ onto $B_{\xi}$. For each element $x$ in $B_{0}$ let $W_{x}=\left\{g_{\xi}(x) \mid \xi<\theta\right\}$ and $V_{x}$ be the set of $c$-condensation points of $W_{x}$ which are in $W_{x}$. $V_{x}$ being a bounded set, there exists a least upper bound to the elements of $V_{x}$, say $v(x)$. For $x \leqq y, v(x) \leqq v(y)$. Since $V_{x}$ has property C, $y<v(x)$ for each element $y$ in $V_{x}$. Let $v(s)$, where $s$ is in $B_{0}$, be an element of the set $S=\left\{v(x) \mid x \in B_{0}\right\}$ which has the following property:

$\left(^{*}\right)$ For each real number $u<v(s)$, there are $2^{N_{0}}$ elements $x, x<s$, in $B_{0}$ such that $v(x)$ is in the half open interval of $R,(u, v(s)]$.

Let $y$ and $t$, where $t<s$, be elements of $V_{s}$ and $B_{0}$ respectively, such that $y<v(t) \leqq v(s)$. By $\left({ }^{*}\right)$ the element $t$ exists. Since $v(t)$ is the least upper bound of the elements of $V_{t}$, there exists an element $z$ in $V_{t}$ such that $y<z<v(t)$. Let

$$
U=\left\{v \mid g_{v}(s) \leqq y\right\} \text { and } V=\left\{v \mid g_{v}(t) \geqq z\right\} .
$$

Since $t<s$ and $y<z$, it follows that $U$ and $V$ are disjoint sets. Since $y$ and $z$ are $c$-condensation points of $V_{s}$ and $V_{t}$ respectively, the power of $U$ and $V$ is $2^{\aleph_{0}}$ each. Let $N=\left\{x \mid t \leqq x \leqq s, x \in B_{0}\right\}, Y=\left\{g_{v}(N) \mid v \in U\right\}, Z=\left\{g_{v}(N) \mid v \in V\right\}$, and $M=Y \cup Z$. Condition (1) is satisfied. From (c) of Lemma 2.2, the power of $N$ is $2^{N_{0}}$. Thus the power of each set $D_{\xi}$ is $2^{N_{0}}$. If $\xi$ is in $U$ and $x$ is in $N$, then $p \leqq g_{\xi}(x) \leqq y$. If $v$ is in $V$ and $x$ is in $N$, then $y<z \leqq g_{v}(x) \leqq q$. Therefore condition (2) is satisfied.

Turning to the proof of Theorem 2.5 let $Y=\left\{P_{\xi} \mid \xi<\theta\right\}$ and $Z=\left\{Q_{v} \mid v<\theta\right\}$ be two families of sets which satisfy the conclusion of Lemma 2.3. Note that $\bar{P}_{0} \leqq \bar{B}_{0}$. Let $h_{\xi}$ and $k_{\xi}$ be similarity transformations of $P_{0}$ onto $P_{\xi}$ and $Q_{\xi}$ respectively. Let $F_{0}$ be an exact subset of $P_{0}$, having property $A$, such that $1+\bar{F}_{0}+1<\bar{P}_{0}$. The set $F_{0}$ exists by Theorems 4 and 5 in [3]. Let $M_{\xi}=h_{\xi}\left(F_{0}\right)$ and $N_{\xi}=k_{\xi}\left(F_{0}\right)$. By means of transfinite induction we can define four disjoint sets, $J_{i}=\left\{p_{\xi}^{i} \mid \xi<\theta\right\}, i<4$, whose set union is the set $E-\left[\cup_{\xi<\theta}\left(M_{\xi} \cup N_{\xi}\right)\right]$, such that

(a) $p_{\xi}^{0}$ precedes, and $p_{\xi}^{1}$ follows, each element of $M_{\xi}$; and

(b) $p_{\xi}^{2}$ precedes, and $p_{\xi}^{3}$ follows, each element of $N_{\xi}$.

In view of (d) of Lemma 2.2 and (2) of Lemma 2.3, the sets $J_{i}, i<4$, exist. Define $T$ to be the family of sets

$$
T=\left\{M_{\xi} \cup\left\{p_{\xi}^{0}, p_{\xi}^{1}\right\} \mid \xi<\theta\right\} \cup\left\{N_{\xi} \cup\left\{p_{\xi}^{2}, p_{\xi}^{3}\right\} \mid \xi<\theta\right\} .
$$

The family $T$ satisfies the conclusion of the theorem. Q.E.D.

Using the methods of this section the following two results can be shown:

Theorem 2.6. Let $D$ be a denumerable set. A necessary and sufficient condition that each set $E$, for which $\bar{E} \equiv \eta$, be the union of a family $\left\{E_{n} \mid n<\omega\right\}$ of pair- 
wise disjoint sets, where $\bar{E}_{n}=\bar{D}$ for each $n$, is that $D$ have both a first and a last element.

THEOREM 2.7. For each sequence of denumerable order types $\left\{\alpha_{n}\right\}_{n<\omega}$, where $\alpha_{n} \neq 0$, the set of rational numbers is the union of a family $\left\{E_{n} \mid n<\omega\right\}$ of pairwise disjoint sets such that $\bar{E}_{n}=\alpha_{n}$.

\section{Decompositions into sets having property $\mathbf{A}$.}

LEMMA 3.1. A linear set $E$, of power $2{ }^{N_{0}}$, which is the union of a family $\left\{A_{i} \mid i \leqq n\right\}$ of pairwise disjoint, similar sets, where $n$ is a non-negative integer, cannot be the union of less than $n+1$ pairwise disjoint sets, each of which has property A.

Proof. Assume the contrary, i.e., assume that $E$ is the union of $k$ disjoint sets $B_{i}$, where $k \leqq n$ and each set $B_{i}$ has property A. Let $f_{i}$ be a similarity transformation of $A_{i}$ onto $A_{i+1}$ for $i<n$. As the power of the set $E$ is $2^{N_{0}}$, the power of each set $A_{i}$ must be $2^{\aleph_{0}}$. Therefore the power of the intersection of at least one of the sets $B_{i}$ with $A_{0}$ is $2^{\mathbb{N}_{0}}$. For simplicity of notation we shall assume that $B_{0}$ is one such set. Denote by $C_{0}$ the set $A_{0}$ and by $C_{1}$ the set $f_{0}\left(A_{0} \cap B_{0}\right)$. Suppose that for each integer $i$, where $1 \leqq i \leqq j$, the set $C_{i}$ of power $2^{N_{0}}$ has been defined so that $C_{i}=f_{i-1}\left(C_{i-1} \cap B_{i-1}\right)$. Now the power of the intersection of at least one of the sets $B_{i}$ with $C_{j}$ is $2{ }^{N_{0}}$. Suppose that for some integer $i<j$ the power of the set $C_{j} \cap B_{i}$ is $2^{\aleph_{0}}$. Then

$$
C_{k} \cap B_{i} \text { and } f_{i}^{*} f_{i+1}^{*} \cdots f_{k-1}^{*}\left(C_{k} \cap B_{i}\right)
$$

are two disjoint, similar subsets of $B_{i}$, of power $2^{N_{0}}$ each. This, however, contradicts the assumption that the set $B_{i}$ has property $\mathrm{A}$. Therefore, if the power of the set $C_{j} \cap B_{i}$ is $2 \mathrm{~N}_{0}$, then $i \geqq j$. For simplicity we assume that the power of the set $C_{j} \cap B_{j}$ is $2^{N_{0}}$. Denote by $C_{j+1}$ the set $f_{j}\left(C_{j} \cap B_{j}\right)$. By mathematical induction, the sets $C_{i}$ become defined for $i \leqq k-1$. Since $k \leqq n$, the set $C_{k}=f_{k-1}\left(C_{k-1} \cap B_{k-1}\right)$ is well defined and of power $2^{N 0}$. Therefore the power of the intersection of at least one of the sets $B_{i}$ with $C_{k}$ is $2^{\text {No }}$. If $B_{i}$ is such a set, where of necessity $i<k$, then

$$
C_{k} \cap B_{i} \text { and } f_{i}^{*} f_{i+1}^{*} \cdots f_{k-1}^{*}\left(C_{k} \cap B_{i}\right)
$$

are two disjoint, similar subsets of $B_{i}$, of power $2^{\aleph_{0}}$ each. This contradicts the assumption that $B_{i}$ has property $\mathrm{A}$. We conclude that $E$ cannot be the union of less than $n+1$ disjoint sets, each of which has property $A$.

Corollary 1. A linear set $E$, of power $2^{\mathrm{N}_{0}}$, whose order type is $\sigma(n+1)$, where $n$ is a non-negative integer, cannot be the union of less than $n+1$ pairwise disjoint sets, each of which has property A.

COROLlaRY 2. $R$ is not the union of a finite number of disjoint sets, each of which has property A. 
TheOREM 3.1. A linear set $E$, which contains a set $D$, of power $2^{\boldsymbol{N}_{0}}$, such that $D$ is the union of a family of $n+1$ pairwise disjoint, similar sets, where $n$ is $a$ non-negative integer, cannot be the union of less than $n+1$ disjoint sets, each of which has property A.

Proof. Suppose that $E$ is the union of a family $\left\{B_{i} \mid i<k\right\}$ of pairwise disjoint sets $B_{i}$, each of which has property $\mathrm{A}$, where $k<n+1$. Let $A_{i}=B_{i} \cap D$. As $D$ is of power $2^{\boldsymbol{N}_{0}}$, at least one of the sets $A_{i}$ is of power $2^{\boldsymbol{N}_{0}}$. Let $C_{0}^{*}$, $C_{1}, \cdots, C_{m}$, where $m<k$, be those sets $A_{i}$ which are of power $2^{\aleph_{0}}$. As subsets, of power $2^{\aleph_{0}}$ each, of sets which have property $\mathrm{A}, C_{0}^{*}$ and $C_{i}$ each has property A. Denote by $C_{0}$ the set union of $C_{0}^{*}$ and those sets $A_{i}$ which are of power $<2^{\aleph_{0}}$. By Lemma 3.1 of [4], $C_{0}$ has property A. This implies that $D$ is the union of less than $n+1$ disjoint sets, each of which has property A. But this contradicts Lemma 3.1. Hence our result.

Corollary. A linear set which contains a set $D$ such that $D$ is the union of a family $\left\{B_{i} \mid i<\omega\right\}$ of pairwise disjoint, similar sets, of power $2^{\boldsymbol{N}_{0}}$ each, cannot be the union of less than $\aleph_{0}$ disjoint sets, each of which has property A.

Open Question. Let $E$ be a linear set of power $2 \boldsymbol{\aleph}_{0}$. If $k$ is the largest integer for which $E$ contains a subset $D$, of power $2^{\aleph_{0}}$, such that $D$ is the union of $k$ pairwise disjoint, similar sets, then is $E$ the union of $k$ disjoint sets, each of which has property $A$ ?

Lемма 3.2. Let $\left\{A_{i} \mid i<n\right\}$ be a finite family of pairwise disjoint, similar, linear sets, of power $2^{\aleph_{0}}$ each. Then there exists a family $\left\{B_{i} \mid i<n\right\}$ of similar, exact sets, with the following properties:

(1) Each set has a first and a last element.

(2) For each $i<n, B_{i}$ is a subset of $A_{i}$.

(3) For each pair of distinct sets $A_{i}$ and $A_{j}$, either $x<y$ for each element $x$ in $B_{i}$ and $y$ in $B_{j}$, or $y<x$ for each element $x$ in $B_{i}$ and $y$ in $B_{j}$.

(4) $B_{i}$ has property A.

Proof. We shall first prove the lemma for $n=2$. Denote by $M$ the set of $c$-condensation points of $A_{0}$ which are in $A_{0}$. The set $M$ has property C. Let $f$ be a similarity transformation of $A_{0}$ onto $A_{1}$. Since $A_{0}$ and $A_{1}$ are disjoint, for any element $p$ in $M, f(p) \neq p$, say $f(p)>p$. As $p$ is a $c$-condensation point of $M$, the power of the set $P=\{x \mid p<x<f(p), x \in M\}$ is $2^{N_{0}}$. The function $f$ being a similarity transformation of $A_{0}$ onto $A_{1}, f(x)>f(p)$ for each element $x$ in $P$. Now $P$ contains an exact subset $B_{0}$, having property $A$, with a first and a last element. Let $B_{1}=f\left(B_{0}\right)$. The two sets, $B_{0}$ and $B_{1}$, satisfy the conclusion of the lemma. An analogous argument occurs if $f(p)<p$.

We continue by induction. Assume that the lemma is true for $n \leqq k$. We now show that it is true for $n=k+1$. By our induction hypothesis there exists a family $\left\{C_{i} \mid i<k\right\}$ of similar, exact sets, having property $\mathrm{A}$, which satisfies 
the lemma. Without loss of generality we may assume that if $x$ is in $C_{i}$ and $y$ is in $C_{j}$, where $i<j<k$, then $x<y$. For each $i<k$, let $a_{i}$ and $b_{i}$ denote the first and the last elements respectively of $C_{i}$. Let $G$ be a subset of $A_{k}$ which is similar to $C_{0}$. Consider the open intervals of $R$,

$$
\left(-\infty, a_{0}\right),\left(a_{0}, b_{0}\right),\left(b_{0}, a_{1}\right), \cdots,\left(a_{k-1}, b_{k-1}\right),\left(b_{k-1},+\infty\right) .
$$

Since the power of $G$ is $2^{\aleph_{0}}$, one of the intervals in $\left(^{*}\right)$ contains $2^{\aleph_{0}}$ elements of $G$.

(a) Suppose that the set $H=G \cap\left(a_{i}, b_{i}\right)$ is of power $2^{\aleph_{0}}$. Since the lemma is true for two sets, $C_{i}$ and $H$ contain exact subsets $B_{i}$ and $B_{k}$ respectively, each of power $2^{\aleph_{0}}$, which satisfy the conclusion of the lemma. For each $j<k, j \neq i$, let $B_{j}$ be a subset of $C_{j}$ which is similar to $B_{i}$.

(b) Suppose that the set $H=G \cap J$, where $J$ is an interval in $\left(^{*}\right)$ not of the form $\left(a_{i}, b_{i}\right)$ for some $i<k$, is of power $2^{\aleph_{0}}$. Let $B_{k}$ be an exact subset of $H$, of power $2^{\aleph_{0}}$, which has a first and a last element. For each $i<k$, let $B_{i}$ be a subset of $C_{i}$ which is similar to $B_{k}$.

In each case the family of sets $\left\{B_{i} \mid i<k+1\right\}$ satisfy the conclusion of the lemma. Q.E.D.

In view of the previous lemma the open question stated prior to it may be phrased as follows: Let $E$ be a linear set of power $2^{\aleph_{0}}$. If $k$ is the largest integer for which $E$ contains a subset $D$, of power $2^{N_{0}}$, such that $\bar{D}=\sigma k$ for some order type $\sigma$, does it follow that $E$ is the union of $k$ disjoint sets, each of which has property $\mathrm{A}$ ? If $\bar{E} \equiv \bar{D}$, then the answer trivially is yes.

We have seen that certain sets cannot be the union of a finite number of disjoint sets, each of which has property A. The problem arises of determining whether or not each linear set, of power $2 \aleph_{0}$, is the union of $\boldsymbol{\aleph}_{0}$ disjoint sets, each of which has property A. The answer to this, in the affirmative provided that one assumes the continuum hypothesis, i.e., $2^{N_{0}}=\boldsymbol{N}_{1}$ (Theorem 3.2). The problem of the decomposition of an arbitrary linear set of power $2^{N_{0}}$, into a family of $2^{\aleph_{0}}$ disjoint sets, each having property A, was settled in the affirmative in Theorem 3.1 of [4].

For each linear set $E$, of power $2^{\aleph_{0}}$, denote by $V(E)$ the family of those Borel subsets of $E$, with respect to $E$, which are of power $2 \aleph_{0}$.

THEOREM 3.2. If $2 \boldsymbol{\aleph}_{0}=\boldsymbol{\aleph}_{1}$, then each linear set $E$, of power $2 \boldsymbol{\aleph}^{\aleph_{0}}$, is the union of a family $\left\{E_{n} \mid n<\omega\right\}$ of pairwise disjoint, exact sets, each of which has property A. In addition, the order types of the $E_{n}$ are pairwise incomparable. If, furthermore, $E$ has property $\mathrm{C}$, then each set $E_{n}$ is a dense subset of $E$, which has property $\mathrm{C}$.

Proof. The demonstration is based, to a certain extent, on a modification of a lemma due to Banach [1].

Denote by $M$ the set of $c$-condensation points of $E$ which belong to $E$. Let $G$ be the set of couples $s=(f, B)$, where $f$ is in $K^{*}(B)$ and $B$ is an element 
of $V(M)$ having property C. Well order the elements of $G$ into the sequence $\left\{s_{\xi}\right\}, \xi<\theta$, where each element in $G$ occurs $2^{\aleph_{0}}$ times in the sequence, and $s_{\xi}=\left(f_{\xi}, B_{\xi}\right)$. We further require the sequence $\left\{s_{\xi}\right\}, \xi<\theta$, to have the property that for $\xi=v+6 t$, where $v$ is either 0 or a limit number and $t$ is a nonnegative integer,

$$
s_{\xi}=s_{\xi+1}=s_{\xi+2}=s_{\xi+3}=s_{\xi+4}=s_{\xi+5} \text {. }
$$

Such a sequence is certainly possible. Denote by $J_{\xi}$ the set

$$
J_{\xi}=\left\{x \mid f_{\xi}(x) \neq x, x \in B_{\xi}\right\} .
$$

Since each element of $B_{\xi}$ is a $c$-condensation point of $B_{\xi}$, the power of $J_{\xi}$ is $2 \aleph_{0}$. Well order the elements of $R$ into the sequence $\left\{x_{\xi}\right\}, \xi<\theta$.

Let $N_{0}^{0}=\left\{p_{i}^{0,0} \mid i<\omega\right\}$ be the set of the first $\omega$ elements in $R$. Let $p_{0}^{0,1}$ be the first element in the set $J_{0}-\left[N_{0}^{0} \cup f_{0}^{*}\left(N_{0}^{0}\right)\right]$, and $p_{0}^{0,2}=f_{0}\left(p_{0}^{0,1}\right)$. In general, for each positive integer $n$, let $p_{n}^{0,1}$ be the first element in the set $J_{0}$ $-\left[N_{n}^{0} \cup f_{0}^{*}\left(N_{n}^{0}\right)\right]$, where

$$
N_{n}^{0}=N_{0}^{0} \cup\left\{p_{i}^{0,1} \mid i<n\right\} \cup\left\{p_{i}^{0,2} \mid i<n\right\}, \quad \text { and } p_{n}^{0,2}=f_{0}\left(p_{n}^{0,1}\right) \text {. }
$$

Denote by $f^{0}$ the identity function and, for $n>0$, by $f^{n}$ the function $f\left[f^{n-1}\right]$, and by $f^{-n}$ the function $f^{*}\left[f^{-n+1}\right]$.

Suppose that for each ordinal number $\xi$, where $\xi<\alpha<\theta$, the set

$$
P^{\xi}=\left\{p_{m}^{\xi, j} \mid j<3, m<\omega\right\}
$$

has been defined. For any finite number of ordinal numbers, $\alpha_{1}, \cdots, \alpha_{k}$, each smaller than $\alpha$, for any integers (positive, negative, or 0 ), $n_{1}, \cdots, n_{k}$, and for any element $x$ in the set $U_{\xi<\alpha} P^{\xi}$, consider the element

$$
f_{\alpha_{1}}^{n_{1}} f_{\alpha_{2}}^{n_{2}} \cdots f_{\alpha_{k}}^{n_{k}}(x) \text {. }
$$

Denote by $W_{\alpha}$ the set of all such elements. Since $2 \boldsymbol{\aleph}_{0}=\boldsymbol{\aleph}_{1}$, the power of the set $W_{\alpha}$ is $\boldsymbol{\aleph}_{0}$. Let $N_{0}^{\alpha}=\left\{p_{i}^{\alpha, 0} \mid i<\omega\right\}$ be the set of the first $\omega$ elements in $R-W_{\alpha}$. Let $p_{0}^{\alpha, 1}$ be the first element in the set $J_{\alpha}-\left[N_{1}^{\alpha} \cup f_{\alpha}^{*}\left(N_{1}^{\alpha}\right)\right]$, where $N_{1}^{\alpha}=N_{0}^{\alpha} \cup W_{\alpha}$, and $p_{0}^{\alpha, 2}=f_{\alpha}\left(p_{0}^{\alpha, 1}\right)$. In general, for each positive integer $n$, let $p_{0}^{\alpha, 1}$ be the first element in the set $J_{\alpha}-\left[N_{n}^{\alpha} \cup f_{\alpha}^{*}\left(N_{n}^{\alpha}\right)\right]$, where

$$
N_{n}^{\alpha}=W_{\alpha} \cup N_{0}^{\alpha} \cup\left\{p_{i}^{\alpha, j} \mid j=1,2 ; i<n\right\},
$$

and $p_{n}^{\alpha, 2}=f_{\alpha}\left(p_{n}^{\alpha, 1}\right)$.

Now denote by $H_{0}$ the set $W_{1}$, and by $H_{v}$ the set $W_{v+1}-W_{v}$. Following the proof of Banach, where $F=\left\{f_{\xi} \mid \xi<\theta\right\}$, we note that

$$
P^{v} \subseteq H_{v} \quad \text { and } \quad\left\{p_{i}^{v, 1} \mid i<\omega\right\} \subseteq H_{v} \cap E .
$$


Since the power of $W_{v+1}$ is $\boldsymbol{\aleph}_{0}$, the power of $H_{v}$, and also of $H_{v} \cap E$, is $\boldsymbol{\aleph}_{0}$. Furthermore, the family of sets $\left\{H_{v} \mid v<\theta\right\}$ satisfy the conclusion of the lemma in [1].

Denote by $\left\{y_{j}^{v} \mid j<q(v) \leqq \omega\right\}$ the elements of the denumerable set

$$
\left(E \cap H_{v}\right)-\left\{p_{j}^{v, i} \mid i=1,2 ; j<\omega\right\} .
$$

We shall now define three families of disjoint subsets of $R,\left\{S_{i}^{*} \mid i<\omega\right\}$, $\left\{T_{i}^{*} \mid i<\omega\right\}$, and $\left\{U_{i}^{*} \mid i<\omega\right\}$, by the following procedure: Let $v$ be 0 or a limit number, and let $t$ be a non-negative integer.

(1) If $\xi=v+6 t$, then $p_{i}^{\xi, 1}$ is in $S_{i}^{*}, p_{i}^{\xi, 2}$ is in $T_{i}^{*}$, and $y_{i}^{\xi}$ is in $U_{i}^{*}$.

(2) If $\xi=v+6 t+1$, then $p_{i}^{\xi, 1}$ is in $S_{i}^{*}, p_{i}^{\xi, 2}$ is in $U_{i}^{*}$, and $y_{i}^{\xi}$ is in $T_{i}^{*}$.

(3) If $\xi=v+6 t+2$, then $p_{i}^{\xi, 1}$ is in $T_{i}^{*}, p_{i}^{\xi, 2}$ is in $S_{i}^{*}$, and $y_{i}^{\xi}$ is in $U_{i}^{*}$.

(4) If $\xi=v+6 t+3$, then $p_{i}^{\xi, 1}$ is in $T_{i}^{*}, p_{i}^{\xi, 2}$ is in $U_{i}^{*}$, and $y_{i}^{\xi}$ is in $S_{i}^{*}$.

(5) If $\xi=v+6 t+4$, then $p_{i}^{\xi, 1}$ is in $U_{i}^{*}, p_{i}^{\xi, 2}$ is in $S_{i}^{*}$, and $y_{i}^{\xi}$ is in $T_{i}^{*}$.

(6) If $\xi=v+6 t+5$, then $p_{i}^{\xi, 1}$ is in $U_{i}^{*}, p_{i}^{\xi, 2}$ is in $T_{i}^{*}$, and $y_{i}^{\xi}$ is in $S_{i}^{*}$.

For each $i$ let $S_{i}=M \cap S_{i}^{*}, T_{i}=T_{i}^{*} \cap M$, and $U_{i}=U_{i}^{*} \cap M$.

Since the family of sets

$$
\left\{S_{i} \mid i<\omega\right\} \cup\left\{T_{i} \mid i<\omega\right\} \cup\left\{U_{i} \mid i<\omega\right\}
$$

is denumerable, its elements may be relabeled as $\left\{Q_{n} \mid n<\omega\right\}$. From the fact that $M$ has property $\mathrm{C}$, it follows that

$(\alpha)$ each set $Q_{n}$ has property C, and

$(\beta)$ each set $Q_{n}$ is a dense subset of $M$.

For each $n$ consider the set $Q_{n}$. Let $Y$ and $Z$ be any two disjoint subsets of $Q_{n}$ of power $2^{\aleph_{0}}$ each. Now let $f$ be any element of $K^{*}(Y)$. The function $f$ can be extended to be an element $g$ of $K^{*}(B)$, where $B$ is an element of $V(M)$. From Theorem 2 of [1], the power of the set $g(Y) \cap Z=f(Y) \cap Z$ is $\left\langle 2^{N_{0}}\right.$. Therefore $Y$ is similar to no subset of $Z$. This shows that $Q_{n}$ has property A. Combining this with $(\alpha)$ we conclude, in virtue of Theorem 2.3 of [4], that $Q_{n}$ is exact. Since $2^{\aleph_{0}}=\boldsymbol{\aleph}_{1}$, the set $E-M$ is denumerable, say $\left\{z_{i} \mid i<\delta \leqq \omega\right\}$. For $i<\delta$ let $E_{i}=Q_{i} \cup\left\{z_{i}\right\}$, and for $i \geqq \delta$, let $E_{i}=Q_{i}$.

From Corollary 2 of Theorem 2 of [3] and Lemma 3.1 of [4], $\left\{E_{n} \mid n<\omega\right\}$ is a family of pairwise disjoint, exact sets, each of which has property $A$. Therefore we only have to show that the order type of the $E_{n}$ are pairwise incomparable. Suppose the contrary, i.e., for two integers $i$ and $j, i \neq j$, there exists a similarity transformation $f$ of $E_{i}$ into $E_{j}$. We shall show that this entails a contradiction. First suppose that either $E_{i}=S_{m} \cup\left\{z_{i}\right\}$ or $E_{i}=S_{m}$. Thus $f$ is a similarity transformation of $S_{m}$ into $E_{j}$. From $(\beta)$, the fact that $E_{i} \cap E_{j}=\varnothing$, and Lemma 1.1 of [4], it follows that $f$, acting on $S_{m}$, can be extended to be an element $g$ in $K^{*}(B)$, where $(g, B)$ is in $G$ and $B$ contains $S_{m}$. From (1), (2), and the properties of the sequence $\left\{s_{\xi}\right\}, \xi<\theta$, there are $2^{\aleph_{0}}$ elements $x$ in $S_{m}$ such that $g(x)$ is in $T_{m}^{*}$, and $2^{\aleph_{0}}$ elements $x$ in $S_{m}$ such that $g(x)$ is in $U_{m}^{*}$. This implies that $f\left(E_{i}\right)$ cannot be a subset of $E_{j}$. If $S_{m}$ is re- 
placed by $T_{m}$ or $U_{m}$, an analogous argument is possible. Under no circumstances, therefore, can $f$ be a similarity transformation of $E_{i}$ into $E_{j}$.

The last sentence in the statement of the theorem is obvious since then $E=M$. Q.E.D.

While proving Theorem 3.2 we have also demonstrated the following result:

THEOREM 3.3. Let $E$ be a linear set of power $2 \aleph_{0}$, and $F$ a family of one-to-one transformations, of power $2 \aleph_{0}$, which contains $K(E)$. If $2^{\aleph_{0}}=\aleph_{1}$, then there exists a family $T=\left\{H_{\xi} \mid \xi<\theta\right\}$ of pairwise disjoint sets which satisfy the following conditions:

(1) $R=\bigcup_{\xi<\theta} H_{\xi}$;

(2) for each ordinal number $\xi, \xi<\theta$, the power of each of the two sets, $H_{\xi}$ and $H_{\xi} \cap E$, is $\boldsymbol{\aleph}_{0}$; and

(3) $f$ being any transformation belonging to $F$ and $D$ being the domain of $f$, there exists an ordinal number $\alpha<\theta$ such that $f\left(D \cap H_{\xi}\right)$ is a subset of $H_{\xi}$ for $\xi>\alpha$.

If, furthermore, $E$ has property $\mathrm{C}$, then the family $T$ can be chosen to satisfy the following additional condition:

(4) for each $\xi<\theta$, if $p_{\xi}$ is an element of $H_{\xi} \cap E$, then the set $D=\left\{p_{\xi} \mid \xi<\theta\right\}$ is a dense subset of $E$ having property $\mathrm{C}$.

Turning to decompositions into a family of $2^{\aleph_{0}}$ sets we have

THEOREM 3.4. Each linear set $E$, of power $2^{\aleph_{0}}$, is the union of a family $H=\left\{E_{\xi} \mid \xi<\theta\right\}$ of pairwise disjoint, exact sets, with the following property: If $P$ is any subfamily of $H$, of power $\left\langle 2^{\aleph_{0}}\right.$, and if $S(P)=\bigcup_{E_{\xi} \in P} E_{\xi}$, then $S(P)$ has property A. Furthermore, if $E$ has property $\mathrm{C}$, then each set $S(P)$ is exact.

Proof. Let $B$ be a subset of $E$ which has properties $A$ and $C$. Let $\left\{x_{\xi} \mid \xi<\mu \leqq \theta\right\}$ be the set $E-B$. Let $B$ be the union of a family $\left\{C_{\xi} \mid \xi<\theta\right\}$ of pairwise disjoint, nonempty sets, each of which has property $C$. Such a decomposition is possible. For example, let $F$ be the family of those open intervals of $R$ which contain at least one point (thus $2^{N_{0}}$ points) of $B$. Let $G$ be the set of those pairs $(I, \xi)$, where $I$ is in $F$ and $\xi$ is an ordinal number $<\theta$. Well order the elements of $G$ into a sequence $\left\{\left(I_{v}, \xi_{v}\right)\right\}$, where each pair in $G$ occurs $2^{\aleph_{0}}$ times in the sequence. Let $B=\left\{y_{\xi} \mid \xi<\theta\right\}$. Let $z_{0}$ be the first element in $I_{0} \cap B$. Continuing by transfinite induction, suppose that $z_{\mu}$ is defined for each $\mu<\gamma$. Let $z_{\gamma}$ be the first element in $\left(I_{\gamma} \cap B\right)-\left\{z_{\mu} \mid \mu<\gamma\right\}$. For each $v<\theta$, let $C_{v}=\left\{z_{\mu} \mid \xi_{\mu}=v\right\}$. It is easily seen that (1) $B=\left\{z_{\gamma} \mid \gamma<\theta\right\}$, (2) $B$ is the union of the disjoint sets $C_{v}$, and (3) each $C_{v}$ has property C. By Theorem 2.3 of [4], each set $C_{\xi}$ is exact. For each $\xi<\mu$ let $E_{\xi}=C_{\xi} \cup\left\{x_{\xi}\right\}$, and for $\xi \geqq \mu$ let $E_{\xi}=C_{\xi}$. Each set $E_{\xi}$ is exact. By Lemma 3.1 of [4], if $P$ is any subfamily of $H$, of power $<2^{\aleph_{0}}$, then $S(P)$ has property A.

On assuming that $E$ has property $\mathrm{C}, B$ and each set $E$ may be chosen to 
be a dense subset of $E$. Therefore each point of $S(P)$ is a c-condensation point of $S(P)$. Since $S(P)$ also has property A, by Theorem 2.3 of $[4], S(P)$ is exact.

REMARK. Let $P$ and $Q$ be any two disjoint subfamilies of $H$, of power $<2^{\text {so }_{0}}$ each. Then $S(P \cup Q)$ has property A. Consequently $S(P)$ and $S(Q)$ have incomparable order types. Letting $P=\left\{E_{\xi}\right\}$ and $Q=\left\{E_{v}\right\}$, i.e., $P$ and $Q$ each consist of just one set, we see that the order types of the $E_{\xi}$ are pairwise incomparable.

In view of the preceding remark we have

COROLlARY. Each linear set $E$, of power $2^{N_{0}}$, is the union of a family $H$ $=\left\{E_{\xi} \mid \xi<\theta\right\}$ of pairwise disjoint, exact sets, with the following two properties:

(1) For each $E_{\xi}$, no two disjoint subsets, of power $2^{\aleph_{0}}$ each, of $E_{\xi}$ are similar.

(2) For $\xi \neq v$, no subset of $E_{\xi}$, of power $2^{\aleph_{0}}$, is similar to a subset of $E_{v}$.

The question arises as to whether or not the above corollary holds for a decomposition into a denumerable family. The answer to this will be shown to be in the negative.

TheOREM 3.5. Let $E$ be a linear set of power $2^{\aleph_{0}}$. For each ordinal number $\alpha$, where $2 \leqq \alpha \leqq \theta, E$ is the union of a family $\left\{E_{\gamma} \mid \gamma<\alpha\right\}$ of pairwise disjoint sets, of power $2^{\aleph_{0}}$ each, with the following property: For each $\gamma<\alpha$, each subset $N$ of power $2^{N_{0}}$ of $E_{\gamma}$, and each similarity transformation $f$ of $N$ into $R$, the power of $[E \cap f(N)]-E_{\gamma}$ is $<2 \aleph^{\aleph_{0}}$.

Proof. Denote by $G$ the set of triples $s=(f, B, v)$, where $B$ is in $V(E), f$ is an element of $K^{*}(B)$ such that the power of the set $\{x \mid f(x) \neq x, x \in B\}$ is $2^{N_{0}}$, and $v$ is an ordinal number $<\alpha$. Well order the elements of $G$ into a sequence $\left\{s_{\xi}\right\}, \xi<\theta$, where $s_{\xi}=\left(f_{\xi}, B_{\xi}, v_{\xi}\right)$. Repeat the procedure of Theorem 3.2, obtaining the sets $W_{v}$ and $H_{v}$. Thus $E$ is the union of the disjoint sets $H_{v} \cap E$, and for each $v, f_{v}\left(H_{\xi} \cap B_{v}\right)$ is a subset of $H_{\xi}$ for $\xi>v$. Notice that since we are not assuming that $2^{\aleph_{0}}=\aleph_{1}$, we can only deduce that the powers of $W_{v}, H_{v}$, $H_{v} \cap E$, and $U_{v<\mu<\theta} H_{v}$ are infinite and $<2^{\aleph_{0}}$. For each $\gamma<\alpha$ let $T(\gamma)$ $=\left\{\xi \mid \gamma=v_{\xi}\right\}$. The $T(\gamma)$ are pairwise disjoint sets, which contain $2^{\aleph_{0}}$ ordinal numbers. For each $\gamma<\alpha$ let $E_{\gamma}=\bigcup_{\xi \in T(\gamma)}\left(H_{\xi} \cap E\right)$. Then $E$ is the union of the family $\left\{E_{\gamma} \mid \gamma<\alpha\right\}$ of pairwise disjoint sets, of power $2^{N_{0}}$ each.

To see that the $E_{\gamma}$ satisfy the theorem let $\gamma$ be any ordinal number $<\alpha$, $N$ a subset of power $2^{N_{0}}$ of $E_{\gamma}$, and $f$ a similarity transformation of $N$ into $R$. Suppose that the power of $[E \cap f(N)]-E_{\gamma}$ is $2^{\aleph_{0}}$. By Lemma 1.1 of [4], the function $f$ can be extended to become an element $f$ of $K^{*}(B)$, for some $B$ in $V\left(E_{\gamma}\right)$, which contains $N$. Since

$$
[f(N) \cap E]-E_{\gamma} \subseteq\{f(x) \mid f(x) \neq x, x \in B\}
$$

it follows from the power assumption on $f$ that the power of $[f(N) \cap E]-E_{\boldsymbol{\gamma}}$ is $2^{\aleph_{0}}$. Therefore there exists a $\delta=\delta_{\gamma}$ such that $f=f_{\delta}$ and $B=B_{\delta}$. Then 


$$
f(N)=f_{\delta}(N)=f_{\delta}\left[\bigcup_{\xi \in T(\gamma)}\left(H_{\xi} \cap N\right)\right]=\underset{\xi \in T(\gamma)}{\bigcup} f_{\delta}\left(H_{\xi} \cap N\right) .
$$

Now $f_{\delta}\left(H_{\xi} \cap N\right)$ is a subset of $H_{\xi}$ for $\xi>\delta$, and $H_{\xi} \cap H_{v}=\varnothing$ for $\xi \neq v$. Hence for each $\xi$ in $T(\gamma)$ which is $>\delta$,

$$
\begin{aligned}
f_{\delta}\left(H_{\xi} \cap N\right) \cap\left(E-E_{\gamma}\right) & =f_{\delta}\left(H_{\xi} \cap N\right) \cap\left[\bigcup_{v \notin T(\gamma)}\left(H_{v} \cap E\right)\right] \\
& =\bigcup_{v \notin T(\gamma)}\left[f_{\delta}\left(H_{\xi} \cap N\right) \cap\left(H_{v} \cap E\right)\right]=\varnothing .
\end{aligned}
$$

Let $S(\gamma)$ be the set of those $\xi$ in $T(\gamma)$ which are $\leqq \delta$. Then

$$
\begin{aligned}
f(N) \cap\left(E-E_{\gamma}\right) & =f_{\delta}(N) \cap\left(E-E_{\gamma}\right)=f_{\delta}\left[\bigcup_{\xi \in T(\gamma)}\left(H_{\xi} \cap N\right)\right] \cap\left(E-E_{\gamma}\right) \\
& =\left[\bigcup_{\xi \in T(\gamma)} f_{\delta}\left(H_{\xi} \cap N\right) \cap\left(E-E_{\gamma}\right)\right] \\
& =\underset{\xi \in T(\gamma)}{\bigcup}\left[f_{\delta}\left(H_{\xi} \cap N\right) \cap\left(E-E_{\gamma}\right)\right] \\
& =\underset{\xi \in T(\gamma)}{\bigcup}\left[f_{\delta}\left(H_{\xi} \cap N\right) \cap\left(E-E_{\gamma}\right)\right] .
\end{aligned}
$$

Since the power of $U_{\xi \leqq \delta} H_{\xi}$ is $<2^{\aleph_{0}}$, the power of $f(N) \cap\left(E-E_{\gamma}\right)=[E \cap f(N)]$ $-E_{\gamma}$ is $\left\langle 2^{\aleph_{0}}\right.$. From this contradiction we conclude that no such function $f$ can exist. Q.E.D.

From Theorem 3.5 there immediately follows

COROLlaRy 1. Let $E$ be a linear set of power $2^{\aleph_{0}}$. For each ordinal number $\alpha$, where $2 \leqq \alpha \leqq \theta, E$ is the union of a family $\left\{E_{\xi} \mid \xi<\alpha\right\}$ of pairwise disjoint sets, each of power $2^{\mathrm{N}_{0}}$, with the following property: For $\xi \neq v$, there is no subset of $E_{\xi}$, of power $2^{\aleph_{0}}$, which is similar to a subset of $E_{v}$.

In Theorem 3.5 let $R=E$ and consider the sets $E_{v}$ obtained. For each $v$ let $P_{v}=R-E_{v}$. On assuming that the order type of one of the two sets $E_{v}$ or $P_{v}$ is $\equiv \lambda$, we see that there exists a similarity transformation $f$ which maps the other set into it. If $\bar{E}_{v} \equiv \lambda$, then $f\left(E_{\gamma}\right) \subseteq E_{v}$, where $\gamma \neq v$, a contradiction of $f\left(E_{\gamma}\right)-E_{\gamma}$ being of power $<2^{\aleph_{0}}$. If $\bar{P}_{v} \equiv \lambda$, then $f\left(E_{v}\right) \subseteq P_{v}$, a contradiction of $f\left(E_{v}\right)-E_{v}$ being of power $<2 \aleph^{\aleph_{0}}$. It follows that both $\bar{E}_{v}$ and $\bar{P}_{v}$ are each $<\lambda$. Therefore, both $E_{v}$ and $P_{v}$, which is the complement of $E_{v}$, contain no perfect set. Another way of expressing this is to say that both $E_{v}$ and its complement meet every perfect set. Since each measurable set of positive measure contains a perfect set, it follows that both $P_{v}$ and $E_{v}$ are nonmeasurable. Summarizing we have

COROLlaRY 2. For each ordinal number $\alpha$, where $2 \leqq \alpha \leqq \theta, R$ is the union of 
a family $\left\{E_{\xi} \mid \xi<\alpha\right\}$ of pairwise disjoint sets with the following property:

For each set $E_{v}$ and each similarity transformation $f$ of $E_{v}$ into $R$, the power of the set $f\left(E_{v}\right)-E_{v}$ is $<2 \boldsymbol{N}_{0}$.

In any such family of sets, each set is necessarily nonmeasurable.

Since each translation of a linear set is also a similarity transformation of that set we have generalized the following two results due to Sierpiński [9, pp. 24-25; 13]:

(a) There exists a linear, nonmeasurable set, of power $2^{\aleph_{0}}$, which is transformed by each translation into itself, with the exception of a set of power $<2 \boldsymbol{\aleph}^{\aleph_{0}}$.

(b) There exists a decomposition of the line into $2^{\aleph_{0}}$ disjoint sets, each of power $2^{\aleph_{0}}$, such that each translation of the line maps each of the sets into itself, with the exception for each set, of a set of power $<2 \mathrm{~N}$.

In Corollary 2 let $\alpha \leqq \omega$ and consider the sets $E_{i}$ which are obtained. Let $\left\{f_{n}\right\}, n<\omega$, be a sequence of similarity transformations of $R$ onto $R$. For a given integer $i$ consider the two sets, $S=\bigcup_{n}\left[R-f_{n}\left(E_{i}\right)\right]$ and $T=U_{n} f_{n}\left(E_{i}\right)$. Suppose that $\bar{T} \equiv \lambda$. Then there exists a similarity transformation $f$ of $P_{i}$ into $T$, where $P_{i}=R-E_{i}$. Since $f\left(P_{i}\right) \subseteq T$,

$$
f\left(P_{i}\right) \cap T=f\left(P_{i}\right) \cap\left[\bigcup_{n} f_{n}\left(E_{i}\right)\right]=\bigcup_{n}\left[f\left(P_{i}\right) \cap f_{n}\left(E_{i}\right)\right]
$$

is of power $2^{\aleph_{0}}$. By König's theorem, for some integer, say $j$, the power of $f\left(P_{i}\right) \cap f_{j}\left(E_{i}\right)$ is $2^{\aleph_{0}}$. In view of $f_{j}$ being a similarity transformation, the subset $N=f_{j}^{*}\left[f\left(P_{i}\right) \cap f_{j}\left(E_{i}\right)\right]$ of $E_{i}$ is of power $2^{\aleph_{0}}$. Furthermore, $N$ is similar to the subset of $f^{*} f_{j}(N)$ of $P_{i}$. This contradicts the fact, obtained from Corollary 2 , that the power of $f^{*} f_{j}(N)-E_{i}$ is $<2^{\aleph_{0}}$. Therefore $\bar{T}<\lambda$. Suppose that $\bar{S} \equiv \lambda$. There exists a similarity transformation $g$ of $E_{i}$ into $S$. Since $g\left(E_{i}\right) \subseteq S$, for some integer, say $k, g\left(E_{i}\right) \cap\left[R-f_{k}\left(E_{i}\right)\right]$ is of power $2^{N_{0}}$. By Corollary 2, since $f_{k}^{*} g$ is a similarity transformation of $E_{i}, f_{k}^{*} g\left(E_{i}\right) \cap\left[R-E_{i}\right]$ is of power $<2^{\aleph_{0}}$. Since $f_{k}^{*}$ is a similarity transformation of $R$ onto $R$ and $g\left(E_{i}\right)$ $\cap\left[R-f_{k}\left(E_{i}\right)\right]$ is of power $2^{\boldsymbol{N}_{0}}$, the power of $f_{k}^{*}\left\{g\left(E_{i}\right) \cap\left[R-f_{k}\left(E_{i}\right)\right]\right\}$ is of power $2^{\aleph_{0}}$. Now

$$
\begin{aligned}
f_{k}^{*}\left\{g\left(E_{i}\right) \cap\left[R-f_{k}\left(E_{i}\right)\right]\right\} & =f_{k}^{*} g\left(E_{i}\right) \cap f_{k}^{*}\left[R-f_{k}\left(E_{i}\right)\right] \\
& =f_{k}^{*} g\left(E_{i}\right) \cap\left[f_{k}^{*}(R)-f_{k}^{*} f_{k}\left(E_{i}\right)\right] \\
& =f_{k}^{*} g\left(E_{i}\right) \cap\left[R-E_{i}\right] .
\end{aligned}
$$

Thus the power of $f_{k}^{*} g\left(E_{i}\right) \cap\left[R-E_{i}\right]$ is $2^{\aleph_{0}}$, a contradiction. We conclude that $\bar{S}<\lambda$. Since $\bar{S}$ and $\bar{T}$ are each $<\lambda$, for every $n, \overline{f_{n}\left(E_{i}\right)}$ and $\overline{R-f_{n}\left(E_{i}\right)}$ are each $<\lambda$. For a linear set $B$, if $\bar{B}<\lambda$, then $B$ contains no perfect set, $B$ therefore being of interior measure zero. Hence $S, T, f_{n}\left(E_{i}\right)$, and $R-f_{n}\left(E_{i}\right)$ are each of interior measure zero. As in the proof of Corollary 2 we see that $f_{n}\left(E_{i}\right)$ and $R-f_{n}\left(E_{i}\right)$ are nonmeasurable. Therefore the exterior measures of 
$f_{n}\left(E_{i}\right)$ and $R-f_{n}\left(E_{i}\right)$ are each positive. Consequently the exterior measures of $S$ and $T$ are each positive. From this it follows that $S$ and $T$ are each nonmeasurable. We have thus shown

CoROLlaRy 3. For each ordinal number $\alpha$, where $2 \leqq \alpha \leqq \omega, R$ is the union of a family $\left\{E_{i} \mid i<\alpha\right\}$ of nonmeasurable, pairwise disjoint sets, of power $2^{\aleph_{0}}$ each, satisfying the following:

For each sequence $\left\{f_{n}\right\}, n<\omega$, of similarity transformations of $R$ onto $R$, each of the two sets $U_{n} f_{n}\left(E_{i}\right)$ and $U_{n}\left[R-f_{n}\left(E_{i}\right)\right]$ is nonmeasurable and of interior measure zero. Furthermore, the order types of each of the two sets are $<\lambda$.

From Corollary 3 we obtain the following result due to Ruziewicz [8] and Sierpiński $[10 ; 11]$ :

"There exists a linear, nonmeasurable set $C$, such that for any sequence of linear sets $\left\{C_{n}\right\}, n<\omega$, each of which is similar to $C$ by a translation, each of the two sets $\cup_{n} C_{n}$ and $\mathrm{U}_{n}\left(R-C_{n}\right)$ is of interior measure zero."

Note that the cited proposition is no longer true if the words "by a translation" are removed. For if $C$ is any linear set, then $C$ is similar to a subset $C_{0}$ of $(0,1)$. Then $R-C_{0}$ is not of interior measure zero, so that $\bigcup_{n}\left(R-C_{n}\right)$ is not of interior measure zero.

Remarks. (1) If $E$ has property C, then the sets $E_{\xi}$ in Theorem 3.5 can be chosen so as to have property C. If, in addition, $E$ is a dense subset of $R$, then each $E_{v}$ can be chosen to be a dense subset of $R$.

(2) Theorem 3.5 cannot be extended to require that at least one of the sets $E_{v}$ have property A. For example, let $R=E$ be the union of a family $\left\{E_{v} \mid v<\alpha\right\}$ of pairwise disjoint sets satisfying Theorem 3.5. Let $f(x)=x+1$. Since the power of $f\left(E_{v}\right)-E_{v}$ is $\left\langle 2^{\aleph_{0}}\right.$, the power of $M_{v}=\left\{x \mid x \in E_{v}, f(x) \in E_{v}\right\}$ is of power $2^{\aleph_{0}}$. Let $z_{v}$ be a $c$-condensation point of $M_{v}$ and let $N_{v}=M_{v}$ $\cap\left(z_{v}-1 / 4, z_{v}+1 / 4\right)$. Then $N_{v}$ and $f\left(N_{v}\right)$ are two disjoint, similar subsets, of power $2^{\aleph_{0}}$ each, of $E_{v}$.

For $\alpha=\theta$, Corollary 1 can be extended to require each of the sets $E_{v}$ to have property A (Corollary of Theorem 3.4). For $\alpha \leqq \omega$, Corollary 1 cannot be extended to require at least one of the sets $E_{v}$ to have property A. For example, let $R=E$. In view of Corollary 2 of Lemma 3.1, only the case where $\alpha=\omega$ needs to be examined. Suppose that $R$ is the union of a family $\left\{E_{i} \mid i<\omega\right\}$ of pairwise disjoint sets which satisfy the conclusion of Corollary 1 . For any integer $i$ let $p$ be an element of $E_{i}$ which is a $c$-condensation point of $E_{i}$. Let $(a, b)$ be an open interval of $R$ which contains the element $p$, and let $G=E_{i}$ $\cap(a, b)$. Since $p$ is a $c$-condensation point of $E_{i}$, the power of the set $G$ is $2^{\aleph_{0}}$. Let $c$ be an element of $R$ which is greater than $b$, and $f$ a similarity transformation of $G$ into the open interval $(b, c)$ of $R$. Since the power of the set $f(G)$ is $2 \aleph^{\aleph_{0}}$, by König's Theorem, for some $j$ the power of the set $E_{j} \cap f(G)$ is $2^{\aleph_{0}}$. However, since the sets $E_{k}$ satisfy the conclusion of Theorem $3.5, j$ must be $i$. This implies that $E_{i}$ contains two similar, disjoint sets, $f(G) \cap E_{\boldsymbol{i}}$ 
and $f^{*}\left(E_{i} \cap f(G)\right)$, of power $2^{\aleph_{0}}$ each. Consequently no set $E_{i}$ can have property A.

(3) Let $E$ be a linear set, of power $2^{\aleph_{0}}$, such that for each element $f$ in $K(E)$, the power of the set $f(E)-E$ is $\left\langle 2^{\aleph_{0}}\right.$. Now each linear set of positive inner measure contains a perfect set, so that its order type is $\equiv \lambda$. Consequently the complement of the set $E$ cannot have positive inner measure. Thus $E$ cannot have finite measure. In [9, pp. 22-24] the following result was proved:

"If $2 \boldsymbol{\aleph}_{0}=\boldsymbol{\aleph}_{1}$, then there exists a linear, nondenumerable set $E$, of measure 0 , which is transformed by each translation, into itself, with the exception of at most a denumerable number of points."

From our discussion it is clear that the word "translation" cannot be replaced by the words "element of $K(E)$."

(4) The problem of whether or not in Theorem 3.5 and Corollary 1 the sets $E_{i}$ can be chosen so that at least one of them contains a fixed point remains open.

\section{Problem $P$ and incomparable order types.}

THEOREM 4.1. Let $\left\{L_{\xi}\right\}, \xi<\theta$, be a sequence of linear sets such that $\bar{L}_{\xi}<\lambda$. Then there exists a sequence $\left\{B_{\xi}\right\}, \xi<\theta$, of sets with the following properties:

(1) $B_{\xi}$ is an exact, dense subset of $R$, which has property $C$.

(2) $L_{\xi}$ is a subset of $B_{\xi}$ such that $\bar{L}_{\xi}<\bar{B}_{\xi}<\lambda$.

(3) If $f$ is any similarity transformation of $B_{\xi}$ into $R$, then for $v \neq \xi$, the power of the set $f\left(B_{\xi}\right)-B_{v}$ is $2^{N_{0}}$ (thus the order types of the $B_{\xi}$ are pairwise incomparable). If $f$ is not the identity, then the power of the set $f\left(B_{\xi}\right)-B_{\xi}$ is $2^{N_{0}}$.

Proof. It follows from Theorem 2.4 of [4] that for each $\xi<\theta$, an exact, dense subset $A_{\xi}$ of $R$, having property $\mathrm{C}$ and containing $L_{\xi}$, can be found. Furthermore, $\bar{L}_{\xi}<\bar{A}_{\xi}<\lambda$.

For simplicity the proof is divided into two parts. We shall first show that there exists a linear set $M_{0}$ whose order type is incomparable with each $\bar{A}_{\xi}$. Furthermore, the set $B_{0}=A_{0} \cup M_{0}$ satisfies (1) and (2) above, and also (4) below.

(4) For any similarity transformation $f$ of $B_{0}$ into $R$ and each $\xi \geqq 1$, the power of the set $f\left(B_{0}\right)-A_{\xi}$ is $2^{N_{0}}$.

To see this let

$$
\begin{aligned}
& T=\left\{(C, f, v) \mid v<\theta, C \in V\left(A_{\xi}\right), \xi<\theta, f \in K(C)\right\}, \\
& U=\left\{(R, f, v) \mid v<\theta, f \in K^{*}(R)\right\},
\end{aligned}
$$

and $S=T \cup U$. The power of $S$ is $2^{N_{0}}$. Well order the elements of $R$ and $S$ into the two sequences, $\left\{x_{\xi}\right\}, \xi<\theta$, and $\left\{s_{\xi}\right\}, \xi<\theta$, where $s_{\xi}=\left(C_{\xi}, f_{\xi}, v_{\xi}\right)$. For each $v<\theta$ such that $C_{v}=R$, denote by $J_{v}$ the set

$$
J_{v}=\left\{x \mid f_{v}(x) \neq x, x \in R\right\} .
$$


Since $f_{v}$ is in $K^{*}(R)$, the set $J_{v}$ contains an interval of $R$, so that $\bar{J}_{v} \equiv \lambda$.

Suppose that $p_{\xi}, q_{\xi}, r_{\xi}$, and $t_{\xi}$ have been defined for $\xi<\gamma<\theta$. Define $P_{\gamma}$ to be the set $\left\{p_{\xi}, q_{\xi}, r_{\xi}, t_{\xi} \mid \xi<\gamma\right\}$. Suppose that $s_{\gamma}$ is in $T$. Denote by $p_{\gamma}$ the first element in the set $E_{\gamma}=\left[R-f_{\gamma}\left(C_{\gamma}\right)\right]-P_{\gamma}$. Since $\bar{C}_{\gamma}<\lambda, \overline{f_{\gamma}\left(C_{\gamma}\right)}<\lambda$. Therefore the power of the set $R-f_{\gamma}\left(C_{\gamma}\right)$ is $2^{N_{0}}$, by Lemma 3 of [14]. Hence the power of the set $E_{\gamma}$ is $2^{N_{0}}$, so that the element $p_{\gamma}$ exists. Let $q_{\gamma}$ and $r_{\gamma}$ represent no elements. Denote by $t_{\gamma}$ the first element in the set

$$
D_{\gamma}=f_{\gamma}\left(C_{\gamma}\right)-\left[P_{\gamma} \cup\left\{p_{\gamma}\right\}\right] .
$$

The element $t_{\gamma}$ certainly exists since the power of $f_{\gamma}\left(C_{\gamma}\right)$ is $2^{N_{0}}$. Suppose that $s_{\gamma}$ is in $U$. Let $p_{\gamma}$ and $t_{\gamma}$ represent no elements. Let $r_{\gamma}$ be the first element in the set $\left[J_{\gamma}-f_{\gamma}^{*}\left(Q_{\gamma}\right)\right]-P_{\gamma}$, where $Q_{\gamma}=A_{0} \cup P_{\gamma}$, and let $q_{\gamma}=f_{\gamma}\left(r_{\gamma}\right)$. Since $\bar{A}_{0}<\lambda, \bar{Q}_{\gamma}<\lambda$ and $\bar{f}_{\gamma}^{*}\left(Q_{\gamma}\right)<\lambda$. Thus the power of the set $\left[J_{\gamma}-f_{\gamma}^{*}\left(Q_{\gamma}\right)\right]$ is $2^{\aleph_{0}}$, so that $r_{\gamma}$ and $q_{\gamma}$ exist. Note that $q_{\gamma}$ is not an element of $Q_{\gamma} \cup\left\{r_{\gamma}\right\}$.

For each $v<\theta$ let $M_{0}^{0}=\left\{p_{\xi}, r_{\xi} \mid s_{\xi}=(C, f, v)\right\}$ and $M_{0}$ the set union of the $M_{0}^{0}$. We shall now show that $\bar{M}_{0}^{v}$ is incomparable with each $\bar{A}_{\xi}$. Let $f$ be a similarity transformation of $A_{\xi}$ into $R$. Since $A_{\xi}$ is an element of $V\left(A_{\xi}\right)$, there exists an ordinal number $\beta$ such that $s_{\beta}=\left(A_{\xi}, f, v\right)$. It follows from the definitions of the element $t_{\beta}$ and the set $M_{0}^{o}$ that $t_{\beta}$ is an element of the set $f\left(A_{\xi}\right)-M_{0}^{0}$. Therefore $f\left(A_{\xi}\right)$ is not a subset of $M_{0}^{0}$, i.e., $f$ is not a similarity transformation of $A_{\xi}$ into $M_{0}^{0}$. Now suppose that $f$ is a similarity transformation which maps $M_{0}^{0}$ into $A_{\xi}$. The function $f^{*}$ is a similarity transformation of $f\left(M_{0}^{v}\right)$ in to $M_{0}^{o}$. By Lemma 1.1 of [4], $f^{*}$ can be extended to be an element $h$ of $K(C)$, for some element $C$, in $V\left(A_{\xi}\right)$, which contains the set $f\left(M_{0}^{v}\right)$. Thus $h[f(x)]=x$ for each element $x$ in the set $M_{0}^{0}$. There exists an ordinal number $\beta$ for which $s_{\beta}=(C, h, v)$. The element $p_{\beta}$ is in the two sets $M_{0}^{o}$ and $R-h(C)$. But

$$
R-h(C) \subseteq R-h\left[f\left(M_{0}^{v}\right)\right]=R-M_{0}^{v} .
$$

Therefore the element $p_{\beta}$ is in the two disjoint sets, $M_{0}^{0}$ and $R-M_{0}^{0}$. From this contradiction it follows that $f$ does not map the set $M_{0}^{0}$ into $A_{\xi}$. Consequently $\bar{M}_{0}^{0}$ and $\bar{A}_{\xi}$ are incomparable order types. A similar procedure shows that $\bar{M}_{0}$ and $\bar{A}_{\xi}$ are incomparable order types. For each $v<\theta, \xi \geqq 1$, and $f$ in $K\left(B_{0}\right), f\left(M_{0}^{0}\right)-A_{\xi}$ is nonempty. Thus the power of the set $f\left(M_{0}\right)-A_{\xi}$ is $2^{N_{0}}$. Therefore the power of the set $f\left(B_{0}\right)-A_{\xi}$ is $2^{\aleph_{0}}$. To see that $B_{0}$ is exact it is sufficient to show that for any element $h$ of $K^{*}\left(B_{0}\right)$ the power of the set $h\left(B_{0}\right)-B_{0}$ is $2 \aleph_{0}$. Since $B_{0}$ contains $A_{0}$ and $A_{0}$ is a dense subset of $R, B_{0}$ is a dense subset of $R$. Thus $h$ can be extended to be an element $f$ of $K^{*}(R)$. For each ordinal number $v$ there exists an ordinal $\beta_{v}$ such that $s_{\beta_{v}}=(R, f, v)$. Since $r_{\beta_{v}}$ is an element of $B_{0}, q_{\beta_{v}}$ is an element of the set $f\left(B_{0}\right)$. Since $q_{\beta_{v}}$ is an element of $R-A_{0}$ which is not in $M_{0}, q_{\beta_{v}}$ is not in $B_{0}$. Therefore the power of the set $f\left(B_{0}\right)-B_{0}$ is $2^{N_{0}}$. Incidentally, the sets $M_{0}$ and $M_{0}^{v}$, being dense subsets of $R$, can be shown to be exact as above (this is not needed for our proof). We shall now assume that for each $\xi<\mu$, where $\mu<\theta$, the linear set $M_{\xi}$ 
has been defined such that $\bar{M}_{\xi}$ is incomparable with each order type $\bar{A}_{\gamma}$, where $\gamma \geqq \mu$. Furthermore, if $B_{\xi}=A_{\xi} \cup M_{\xi}$, then

(5) for any element $f$ in $K^{*}\left(B_{\xi}\right)$ the power of the set $f\left(B_{\xi}\right)-B_{\xi}$ is $2^{N_{0}}$ (thus $B_{\xi}$ is exact);

(6) for any element $f$ of $K\left(B_{\xi}\right)$, where $\xi<\mu$, and each $\gamma \geqq \mu$, the power of the set $f\left(B_{\xi}\right)-A_{\gamma}$ is $2^{\aleph_{0}}$; and

(7) if $\xi<\mu$ and $v<\mu, \xi \neq \mu$, then for each element $f$ in $K\left(B_{\xi}\right)$, the power of $f\left(B_{\xi}\right)-B_{v}$ is $2^{\aleph_{0}}$.

For $\mu=1, M_{\xi}$ has already been defined. We now define $M_{\mu}$. To do this we modify the argument given above. Let

$$
\begin{aligned}
T= & \left\{(C, f, v, D, g) \mid v<\theta ; C \in V\left(A_{\xi}\right), \xi \geqq \mu, f \in K(C) ;\right. \\
& \left.D=B_{\alpha}, \alpha<\mu, g \in K(D)\right\}, \\
U= & \left\{(R, f, v) \mid v<\theta ; f \in K^{*}(R)\right\}, \\
V= & \left\{(C, f, v) \mid v<\theta ; C \in V\left(B_{\alpha}\right), \alpha<\mu, f \in K(C)\right\},
\end{aligned}
$$

and $S=T \cup U \cup V\left({ }^{2}\right)$. The power of $S$ is $2^{N_{0}}$. Well order the elements of $S$ into the sequence $\left\{s_{\xi}\right\}, \xi<\theta$, where $s_{\xi}$ has one of the forms $\left(C_{\xi}, f_{\xi}, v_{\xi}, D_{\xi}, g_{\xi}\right)$, $\left(R, f_{\xi}, v_{\xi}\right)$, or $\left(C_{\xi}, f_{\xi}, v_{\xi}\right)$.

Suppose that $p_{\xi}, q_{\xi}, r_{\xi}, t_{\xi}$, and $u_{\xi}$ have been defined for $\xi<\gamma<\theta$. Define $P_{\gamma}$ to be the set $\left\{p_{\xi}, q_{\xi}, r_{\xi}, t_{\xi}, u_{\xi} \mid \xi<\gamma\right\}$. If $s_{\gamma}$ is in $T$, define $p_{\gamma}, q_{\gamma}, r_{\gamma}$, and $t_{\gamma}$ as in the first case. Let $u_{\gamma}$ be the first element in the set

$$
\left[g_{\gamma}\left(D_{\gamma}\right)-A_{\mu}\right]-\left[P_{\gamma} \cup\left\{p_{\gamma}, q_{\gamma}, r_{\gamma}, t_{\gamma}\right\}\right] .
$$

By (6) the element $u_{\gamma}$ exists. If $s_{\gamma}$ is in $U$, define $p_{\gamma}, q_{\gamma}, r_{\gamma}$, and $t_{\gamma}$ as in the first case. Let $u_{\gamma}$ represent no element. If $s_{\gamma}$ is in $V$, define $p_{\gamma}, q_{\gamma}, r_{\gamma}$, and $t_{\gamma}$ as if $s_{\gamma}$ were in $T$. Let $u_{\gamma}$ represent no element.

For each $v<\theta$ let $M_{\mu}^{0}=\left\{p_{\xi}, r_{\xi} \mid s_{\xi}=(C, f, v, D, g)\right\}$, and $M_{\mu}$ the set union of the $M_{\mu}^{0}$. The argument given in the first case can be applied to show that $\bar{M}_{\mu}$ is incomparable with each order type $\bar{A}_{\gamma}$, where $\gamma>\mu$. Furthermore, if $B_{\mu}=A_{\mu} \cup M_{\mu}$, then

(8) for any element $f$ in $K^{*}\left(B_{\mu}\right)$, the power of the set $f\left(B_{\mu}\right)-B_{\mu}$ is $2^{N_{0}}$;

(9) for any element $f$ of $K\left(B_{\mu}\right)$ and each $\gamma>\mu$, the power of the set $f\left(B_{\mu}\right)-A_{\gamma}$ is $2^{N_{0}}$; and

(10) for any element $g$ of $K\left(B_{\mu}\right)$ and each $\xi<\mu$, the power of the set $g\left(B_{\mu}\right)-B_{\xi}$ is $2^{N_{0}}$.

For each element $g$ of $K\left(B_{\alpha}\right)$, where $\alpha<\mu$, consider the power of the set $g\left(B_{\alpha}\right)-B_{\mu}$. To each $v<\theta$ there corresponds an ordinal number $\alpha_{v}$ such that $s_{\alpha_{v}}=\left(A_{\mu}, I, v, B_{\alpha}, g\right)$, where $I$ is the identity transformation of $A_{\mu}$. Each ele-

(2) To be logically correct a superscript $\mu$ should be affixed on all the letters appearing in the $\mu$ th case to distinguish them from the letters used in the $\alpha$ th case, $\alpha<\mu$. Since no misunderstanding will occur if the superscript is omitted, this is done. The omission of the superscript will also be done in the proof of Theorem 4.3. 
ment $u_{\alpha_{v}}$ is in $g\left(B_{\alpha}\right)-A_{\mu}$. Furthermore, $u_{\alpha_{v}}$ is not in $M_{\mu}$. Thus each element $u_{\alpha_{v}}$ is in $g\left(B_{\alpha}\right)-B_{\mu}$. Therefore the power of $g\left(B_{\alpha}\right)-B_{\mu}$ is $2^{N_{0}}$.

By transfinite induction we obtain a sequence of sets $\left\{B_{\xi}\right\}, \xi<\theta$. This sequence of sets clearly satisfies the conclusion of the theorem. Q.E.D.

The reasoning in Theorem 4.1 has also demonstrated

THEOREM 4.2. Let $\left\{L_{\xi}\right\}, \xi<\theta$, be a sequence of linear sets, of power $2^{\mathbb{N}_{0}}$ each, such that $\bar{L}_{\xi}<\lambda$. Then there exists an exact, linear set $B$, of power $2^{N_{0}}$, whose order type is incomparable with each $\bar{L}_{\xi}$.

A companion result to Theorem 4.1 is

THEOREM 4.3. Let $\left\{L_{\xi}\right\}, \xi<\theta$, be a sequence of linear sets, of power $2^{\aleph_{0}}$ each. Then there exists a sequence of sets $\left\{B_{\xi}\right\}, \xi<\theta$, whose members have the following properties:

$(\alpha) B_{\xi}$ is a subset of $L_{\xi}$ such that $\bar{B}_{\xi}<\bar{L}_{\xi}$;

$(\beta) B_{\xi}$ is an exact set which has properties $A$ and $C$ (thus, if $f$ is an element of $K^{*}\left(B_{\xi}\right)$, the power of the set $f\left(B_{\xi}\right)-B_{\xi}$ is $\left.2^{N_{0}}\right)$; and

$(\gamma)$ if $f$ is any similarity transformation of $B_{\xi}$ into $R$, then for $v \neq \xi \xi$, the power of the set $f\left(B_{\xi}\right)-B_{v}$ is $2^{\aleph_{0}}$ (thus the $\bar{B}_{\xi}$ are pairwise incomparable order types).

Proof. For each $\xi$ let $A_{\xi}$ be a subset of $L_{\xi}$, having properties A and C, such that $\bar{A}_{\xi}<\bar{L}_{\xi}$. For each $\xi$ let $A_{\xi}$ be the union of $2^{\aleph_{0}}$ disjoint sets $A_{\xi}^{\gamma}, \gamma<\theta$, and each $A_{\xi}^{\gamma}$ the union of $2^{\aleph_{0}}$ disjoint sets $A_{\xi}^{\gamma o}, v<\theta$, the power of each set $A_{\xi}^{\gamma o}$ being $2{ }^{N_{0}}$. Let

$$
Q=\left\{A_{\xi} \mid \xi<\theta\right\} \cup\left\{A_{\xi}^{\gamma} \mid \xi, \gamma<\theta\right\} \cup\left\{A_{\xi}^{\gamma v} \mid \xi, \gamma, v<\theta\right\} .
$$

Well order the elements of $Q$ into a sequence

$$
T_{0}, T_{1}, \cdots, T_{\xi}, \cdots
$$

which has the following three properties:

(a) Each set $A_{\mu}^{\gamma}$ follows $A_{\mu}$ in (1).

(b) Each set $A_{\mu}^{\gamma v}$ follows $A_{\mu}^{\gamma}$ in (1).

(c) The sequence $\left\{A_{\xi}\right\}, \xi<\theta$, is a subsequence of $\left\{T_{\xi}\right\}, \xi<\theta$.

Such a well ordering is certainly possible. Note that $T_{0}=A_{0}$.

We shall first show that there exists an exact subset $B_{0}$ of $A_{0}$, having property $\mathrm{C}$, such that for $\xi \geqq 1$ and $f$ any similarity transformation of $T_{\xi}$ into $R$, the power of the set $f\left(T_{\xi}\right)-B_{0}$ is $2 \mathrm{~N}_{0}$.

To see this let $G^{0}=\left\{T_{\xi} \mid \xi>0\right\}$. Let $S^{0}$ be the set of ordered pairs, $(C, f)$, where $C$ is in $G^{0}$ and $f$ is a similarity transformation of $C$ into $R$. The power of $S^{0}$ is $2^{N_{0}}$. Well order the elements of $R$ and $S^{0}$ into the two sequences

$$
\begin{array}{cc}
x_{0}, x_{1}, \cdots, x_{\xi}, \cdots & (\xi<\theta), \\
s_{0}, s_{1}, \cdots, s_{\xi}, \cdots & (\xi<\theta),
\end{array}
$$


where $s_{\xi}=\left(C_{\xi}, f_{\xi}\right)$ and each element of $S^{0}$ appears $2^{\aleph_{0}}$ times in (3). Suppose that $p_{\xi}$ and $q_{\xi}$ have been defined for $\xi<\mu<\theta$. Let

$$
R_{\mu}=\left\{p_{\xi}, q_{\xi} \mid \xi<\mu\right\}, X_{\mu}=f_{\mu}\left(C_{\mu}\right)-A_{0}, \quad \text { and } Y_{\mu}=f_{\mu}\left(C_{\mu}\right)-X_{\mu} .
$$

Notice that $Y_{\mu}$ is a subset of $A_{0}$. If the power of $Y_{\mu}$ is $2^{N_{0}}$, let $p_{\mu}$ be the first element in the set $Y_{\mu}-R_{\mu}$. If the power of $Y_{\mu}$ is $\left\langle 2^{N_{0}}\right.$, then the power of $X_{\mu}$ is $2^{\aleph_{0}}$. In this case let $p_{\mu}$ represent no element. Suppose that $C_{\mu}$ is the set $A_{0}$ and that $f_{\mu}$ is not the identity transformation on $A_{0}$. Since $A_{0}$ has property C, the power of the set $V_{\mu}=\left\{x \mid f_{\mu}(x) \neq x, x \in A_{0}\right\}$ is $2^{\aleph_{0}}$. Let $q_{\mu}$ be the first element in the set $V_{\mu}-P_{\mu}$, where $P_{\mu}=R_{\mu} \cup\left\{p_{\mu}\right\}$. If $C_{\mu}$ is not the set $A_{0}$, or if $C_{\mu}$ is the set $A_{0}$ and $f_{\mu}$ is the identity transformation on $A_{0}$, let $q_{\mu}$ represent no element.

Denote by $M^{0}$ the set $M^{0}=\left\{p_{\xi} \mid \xi<\theta\right\}$, by $N^{0}$ the set $N^{0}=\left\{q_{\xi} \mid \xi<\theta\right\}$, and by $B_{0}$ the set $B_{0}=A_{0}-M^{0}$. Note that $N^{0}$ is a dense subset of $A_{0}$ which has property C. Since $N^{0}$ is a subset of $B_{0}$, it follows that $B_{0}$ is a dense subset of $A_{0}$ which has property $\mathrm{C}$. Being the subset of a set which has property $\mathrm{A}$, the set $B_{0}$ has property A. By Theorem 2.3 of [4], $B_{0}$ is exact. Suppose that the power of the set $f_{\mu}\left(C_{\mu}\right)-A_{0}$ is $\left\langle 2^{\aleph_{0}}\right.$. There exists an increasing sequence of ordinal numbers $\left\{\alpha_{\xi}\right\}, \xi<\theta$, such that $f_{\mu}\left(C_{\mu}\right)=f_{\alpha_{\xi}}\left(C_{\alpha_{\xi}}\right)$. From the definition of $P_{\alpha \xi}$, it follows that the element $p_{\alpha_{\xi}}$ is in the set $f_{\alpha_{\xi}}\left(C_{\alpha_{\xi}}\right)-B_{0}$. Therefore the power of the set $f_{\mu}\left(C_{\mu}\right)-B_{0}$ is $2^{N_{0}}$.

Now suppose that for each $\xi<\mu<\theta$, the set $B_{\xi}$ has been defined, and has the following properties:

(d) $B_{\xi}$ is an exact subset of $A_{\xi}$ which has properties $\mathrm{A}$ and C.

(e) If $f$ is any similarity transformation of $T_{v}$ into $R$, where $T_{v}$ follows $A_{\xi}$ in (1), then the power of the set $f\left(T_{v}\right)-B_{\xi}$ is $2{ }^{N_{0}}$.

(f) If $f$ is any similarity transformation of $B_{\xi}$ into $R$, and if $\xi<\mu$ and $\gamma<\mu$, where $\xi \neq \gamma$, then the power of the set $f\left(B_{\xi}\right)-B_{\gamma}$ is $2^{N_{0}}$.

For $\mu=1, B_{\xi}$ has already been defined. We now define $B_{\mu}$. Suppose that the set $A_{\mu}^{\gamma v}-g(E)$ is empty, where $E$ is some subset of $B_{\xi}, \xi<\mu$, and $g$ is some similarity transformation of $E$ into $R$. Then the set $g^{*}\left(A_{\mu}^{\gamma v}\right)-E$ is empty. Thus the set $g^{*}\left(A_{\mu}^{\gamma \theta}\right)-B_{\xi}$ is empty. Now $A_{\mu}^{\gamma \theta}$ follows $A_{\mu}^{\gamma}$ and $A_{\mu}^{\gamma}$ follows $A_{\mu}$, in (1). Since $\left\{A_{\xi}\right\}, \xi<\theta$, is a subsequence of (1), $A_{\mu}$ follows $A_{\xi}$, in (1). This implies that $A_{\mu}^{\gamma v}$ follows $A_{\xi}$. From the induction hypothesis, $g^{*}\left(A_{\mu}^{\gamma v}\right)-B_{\xi}$ is nonempty. We conclude that the set $A_{\mu}^{\gamma v}-g(E)$ is nonempty. Consequently the power of the set $A_{\mu}^{\gamma}-g(E)$ is $2^{N_{0}}$.

Denote by $F^{\mu}$ the family $F^{\mu}=\left\{W \mid W \in V\left(B_{\xi}\right), \xi<\mu\right\}$. Let

$$
H^{\mu}=\left\{T_{\xi} \mid T_{\xi} \text { follows } A_{\mu} \text { in (1) }\right\} \text {, and } G^{\mu}=F^{\mu} \cup H^{\mu} .
$$

The power of $G^{\mu}$ is $2^{\aleph_{0}}$. Let $S^{\mu}$ be the set of triples $\left(A_{\mu}^{\gamma}, C, f\right)$, where $\gamma<\theta, C$ is in $G^{\mu}$, and $f$ is in $K(C)$. The power of $S^{\mu}$ is $2^{\aleph_{0}}$. Well order the elements of $S^{\mu}$ into the sequence 


$$
s_{0}, s_{1}, \cdots, s_{\xi}, \cdots
$$

where $s_{\xi}=\left(D_{\xi}, C_{\xi}, f_{\xi}\right)$, and each element of $S^{\mu}$ appears $2 \aleph_{0}$ times in (4). Assume that $p_{\xi}, q_{\xi}$, and $r_{\xi}$ have been defined for $\xi<\gamma<\theta$. If $C_{\gamma}$ is an element of $F^{\mu}$, let $r_{\gamma}$ be the first element in the set, $\left[D_{\gamma}-f_{\gamma}\left(C_{\gamma}\right)\right]-R_{\gamma}$, where $R_{\gamma}$ $=\left\{p_{\xi}, q_{\xi}, r_{\xi} \mid \xi<\gamma\right\}$. The element $r_{\gamma}$ certainly exists since $D_{\gamma}$ is one of the sets $A_{\mu}^{0}$, and the power of the set $A_{\mu}^{0}-f_{\gamma}\left(C_{\gamma}\right)$ is $2^{\aleph_{0}}$. If $C_{\gamma}$ is not in $F^{\mu}$, let $r_{\gamma}$ represent no element. Let $X_{\gamma}=f_{\gamma}\left(C_{\gamma}\right)-A_{\mu}$ and $Y_{\gamma}=f_{\gamma}\left(C_{\gamma}\right)-X_{\gamma}$. Notice that $Y_{\gamma}$ is a subset of $A_{\mu}$. If the power of $Y_{\gamma}$ is $2^{\aleph_{0}}$, let $p_{\gamma}$ be the first element in the set $Y_{\gamma}-\left[R_{\gamma} \cup\left\{r_{\gamma}\right\}\right]$. If the power of $Y_{\gamma}$ is $\left\langle 2^{\boldsymbol{N}_{0}}\right.$, then the power of the set $X_{\gamma}$ is $2 \mathrm{~N}^{\mathrm{N}_{0}}$. In this case let $p_{\gamma}$ represent no element. Suppose that $C_{\gamma}$ is the set $A_{\mu}$ and $f_{\gamma}$ is not the identity transformation on $A_{\mu}$. Since $A_{\mu}$ has property $\mathrm{C}$, the power of the set $V_{\gamma}=\left\{x \mid f_{\gamma}(x) \neq x, x \in A_{\mu}\right\}$ is $2^{\aleph_{0}}$. Let $q_{\gamma}$ be the first element in the set $V_{\gamma}-P_{\gamma}$, where $P_{\gamma}=R_{\gamma} \cup\left\{p_{\gamma}, r_{\gamma}\right\}$. If $C_{\gamma}$ is not the set $A_{\mu}$, or if $C_{\gamma}$ is the set $A_{\mu}$, let $q_{\gamma}$ represent no element.

For each ordinal number $\mu<\theta$ let $M^{\mu}=\left\{p_{\xi} \mid \xi<\theta\right\}, N^{\mu}=\left\{q_{\xi} \mid \xi<\theta\right\}, B_{\mu}$ $=A_{\mu}-M^{\mu}$, and $B_{\mu}^{\gamma}=A_{\mu}^{\gamma} \cap B_{\mu}$. Note that $N^{\mu}$ is a dense subset of $A_{\mu}$ which has property C. Since $N^{\mu}$ is a subset of $B_{\mu}, B_{\mu}$ is a dense subset of $A_{\mu}$ which has property C. Condition (d) is satisfied for $\xi \leqq \mu$. It is easily seen that

(5) the power of each of the two sets, $B_{\mu}^{\gamma}$ and $f_{\gamma}\left(C_{\gamma}\right)-B_{\mu}$, where $C_{\gamma}$ is in $G^{\mu}$, is $2^{\aleph_{0}}$. Let $C_{\gamma}$ be an element of $F^{\mu}$. Consider the set $B_{\mu}^{o}-f_{\gamma}\left(C_{\gamma}\right)$. There exists an ordinal number $\beta$ such that $s_{\beta}=\left(A_{\mu}^{0}, C_{\gamma}, f_{\gamma}\right)$. Since $r_{\beta}$ is an element of the two sets, $D_{\beta}-f_{\beta}\left(C_{\beta}\right)$ and $B_{\mu}$, it follows that the set $B_{\mu}^{0}-f_{\gamma}\left(C_{\gamma}\right)$ is nonempty.

Consider the two sets $B_{\xi}$ and $B_{\mu}$, where $\xi<\mu$. If $f$ is a similarity transformation of $B_{\xi}$ into $R$, then, from (5), the power of the set $f\left(B_{\xi}\right)-B_{\mu}$ is $2^{N_{0}}$. Now let $f$ be any similarity transformation of $B_{\mu}$ into $R$. Let $h$ be the function defined by $h(x)=f(x)$ for $x$ in $B_{\mu}^{\gamma}$. Suppose that $h\left(B_{\mu}^{\gamma}\right)$ is a subset of $B_{\xi}$. Then $h^{*}$ can be extended to be a similarity transformation $g$ of $C$ into $R$, for some element $C$, in $V\left(B_{\xi}\right)$, which contains the set $h\left(B_{\mu}^{\gamma}\right)$. Consider the set $B_{\mu}^{\gamma}-g(C)$. There exists an ordinal number $\beta$ such that $s_{\beta}=\left(A_{\mu}^{\gamma}, C, g\right)$. The element $r_{\beta}$ is in $B_{\mu}^{\gamma}-g(C)$. But

$$
B_{\mu}^{\gamma}-g(C) \subseteq B_{\mu}^{\gamma}-g\left[f\left(B_{\mu}^{\gamma}\right)\right]=B_{\mu}^{\gamma}-B_{\mu}^{\gamma}=\varnothing .
$$

From this contradiction we see that $h$ does not map the set $B_{\mu}^{\gamma}$ into $B_{\xi}$, i.e., $f\left(B_{\mu}^{\gamma}\right)-B_{\xi}$ is nonempty. Consequently the power of the set $f\left(B_{\mu}\right)-B_{\xi}$ is $2^{\aleph_{0}}$.

By transfinite induction we define a sequence of sets, $\left\{B_{\xi}\right\}, \xi<\theta$. The members of this sequence satisfy the conclusion of Theorem 4.3.

Theorem 4.2 may be sharpened as follows.

THEOREM 4.4. Let $\left\{L_{\xi}\right\}, \xi<\theta$, be a sequence of linear sets, of power $2^{N_{0}}$ each, such that $\bar{L}_{\xi}<\lambda$. Then there exists an exact set $B$, having property $\mathrm{A}$, whose order type is incomparable with each $\bar{L}_{\xi}$.

Proof. Let $S$ denote the set of all 5-tuples $(I, C, f, D, g)$, where $I$ is any half-open interval of $R, C$ is in $V(R), f$ is in $K(C), D$ is in $V\left(L_{v}\right)$, where 
$v<\theta$, and $g$ is in $K(D)$. Well order the elements of $R$ and $S$ into the two sequences $\left\{x_{\xi}\right\}, \xi<\theta$, and $\left\{s_{\xi}\right\}, \xi<\theta$, where $s_{\xi}=\left(I_{\xi}, C_{\xi}, f_{\xi}, D_{\xi}, g_{\xi}\right)$. Suppose that $p_{\xi}, q_{\xi}$, and $r_{\xi}$ have been defined for each $\xi<\alpha<\theta$. Let $P_{\alpha}=\left\{p_{\xi}, q_{\xi} \mid \xi<\alpha\right\}$ and $Q_{\alpha}=P_{\alpha} \cup\left\{r_{\xi} \mid \xi<\alpha\right\}$. Denote by $p_{\alpha}$ the first element in $R-S_{\alpha}$, where

$$
S_{\alpha}=Q_{\alpha} \cup \bigcup_{\xi<\alpha} f_{\xi}\left(P_{\alpha}\right) \cup \underset{\xi<\alpha}{\cup} f_{\xi}^{*}\left(P_{\alpha}\right) .
$$

Denote by $q_{\alpha}$ the first element in the set $I_{\alpha}-\left[g_{\alpha}\left(D_{\alpha}\right) \cup T_{\alpha}\right]$, where

$$
T_{\alpha}=S_{\alpha} \cup\left\{p_{\alpha}\right\} \cup\left\{f_{\xi}\left(p_{\alpha}\right) \mid \xi<\alpha\right\} \cup\left\{f_{\xi}^{*}\left(p_{\alpha}\right) \mid \xi<\alpha\right\} .
$$

Since $\bar{D}_{\alpha}<\lambda, \overline{g_{\alpha}\left(D_{\alpha}\right)}<\lambda$. By Lemma 3 of [14], the power of $I_{\alpha}-g_{\alpha}\left(D_{\alpha}\right)$ is $2 \aleph_{0}$. Consequently $q_{\alpha}$ exists. Denote by $r_{\alpha}$ the first element of $g_{\alpha}\left(D_{\alpha}\right)$ $-\left[T_{\alpha} \cup\left\{q_{\alpha}\right\}\right]$. Let $B=\left\{p_{\xi}, q_{\xi} \mid \xi<\theta\right\}$.

To show that $B$ has property $A$ we modify the argument given in Theorem 5 of [3]. Suppose that $F$ and $G$ are two disjoint, similar subsets, of power $2^{\aleph_{0}}$ each, of $B$. Let $f$ be a similarity transformation of $F$ onto $G$. By Lemma 1.1 of [4], $f$ may be extended to be an element $f_{\xi}$ of $K\left(C_{\xi}\right)$, for some ordinal number $\xi$, where $C_{\xi}$ is an element of $V(R)$ which contains the set $F$. For each element $p_{\alpha}$ which is in $F$, where $\alpha>\xi$, consider $f_{\xi}\left(p_{\alpha}\right)$. As $f_{\xi}$ maps $F$ onto $G$, $f_{\xi}\left(p_{\alpha}\right) \neq p_{\alpha}$. From the definition of $q_{\alpha}, f_{\xi}\left(p_{\alpha}\right) \neq q_{\alpha}$. For each ordinal number $\tau>0, p_{\alpha+\tau}$ and $q_{\alpha+\tau}$ are not elements of $f_{\xi}\left(P_{\alpha+\tau}\right)$. Since $p_{\alpha}$ is in $P_{\alpha+\tau}, f_{\xi}\left(p_{\alpha}\right)$ $\neq p_{\alpha+\tau}$ and $f_{\xi}\left(p_{\alpha}\right) \neq q_{\alpha+\tau}$. Suppose that $f_{\xi}\left(p_{\alpha}\right)=p_{\mu}$ or that $f_{\xi}\left(p_{\alpha}\right)=q_{\mu}$, where $\mu<\alpha$. Then $f_{\xi}\left(p_{\alpha}\right)$ is an element of $P_{\alpha}$, so that $p_{\alpha}$ is an element of $f_{\xi}^{*}\left(P_{\alpha}\right)$. This, however, contradicts the manner in which $p_{\alpha}$ was selected. Consequently $f_{\xi}\left(p_{\alpha}\right) \neq p_{\mu}$ and $f_{\xi}\left(p_{\alpha}\right) \neq q_{\mu}$, for $\mu<\alpha$. It follows that for $\alpha>\xi$ and $p_{\alpha}$ in $F, f_{\xi}\left(p_{\alpha}\right)$ is not in $B$. An analogous argument shows that for $\alpha>\xi$ and $q_{\alpha}$ in $F, f_{\xi}\left(q_{\alpha}\right)$ is not in $B$. This implies that the power of the set $f_{\xi}(F) \cap B$ is $<2^{N_{0}}$. But

$$
f_{\xi}(F) \cap B=G \cap B=G,
$$

which is of power $2^{N_{0}}$. From this contradiction we conclude that the two sets $F$ and $G$ do not exist, i.e., $B$ has property A.

To show that $\bar{B}$ and $\bar{L}_{\xi}$ are incomparable order types, for each $\xi<\theta$, repeat the argument given in Theorem 4.1, using $q_{\beta}$ and $r_{\beta}$ for $p_{\beta}$ and $t_{\beta}$ respectively.

To show that $B$ is exact we shall show that each point of $B$ is fixed. Let $y$ be any element of $B$ and let $I$ be any half-open interval of $R,(x, y]$ or $[y, z)$. For each $v<\theta, I$ appears in one of the 5 -tuples $\left(I, R, f, L_{v}, g\right)$. Hence for suitable ordinal numbers $\alpha_{\xi}, \xi<\theta, I=I_{\alpha_{\xi}}$. Then $\left\{p_{\alpha_{\xi}} \mid \xi<\theta\right\}$ is a subset of $B \cap I$. Therefore $y$ is a $c$-condensation point of $B$. From Theorem 2.3 of [4], $y$ is a fixed point of $B$. Q.E.D.

We conclude with a generalization of Theorem 1.1 of [4].

TheOREM 4.5. Let $B$ be a subset, of power $2^{\aleph_{0}}$, of the linear set $E$, such that 
$\bar{B}<\bar{E}$. Let $B$ have the property that if $\bar{X} \leqq \bar{B}$, then the power of the set $E-X$ is $2^{N_{0}}$. Then there exists an exact subset $M$ of $E$ such that

(1) $M$ has properties A and C;

(2) $\bar{M}$ and $\bar{B}$ are incomparable order types; and

(3) $\bar{B}<\overline{B \cup M}<\bar{E}$.

If, furthermore, $E$ has property $\mathrm{C}$, then $M$ is a dense subset of $E$. If $E=R$, then $M$ can be defined so that the set $B \cup M$ is exact.

Proof. For each element $D$ in $V(E)$ let $L(D)$ be the set of those similarity transformations $f$ of $D$ into $R$ for which the power of the set $\{x \mid f(x) \neq x$, $x \in D\}$ is $2^{N_{0}}$. Denote by $P$ the set of couples,

$$
P=\{(D, f) \mid D \in V(E), f \in L(D)\} .
$$

Denote by $Q$ the set of all triples $(D, f, g)$, where $D$ is in $V(B), f$ is in $K(D)$, and $g$ is in $K(E)$. The power of the set $S=P \cup Q$ is $2^{N_{0}}$. Well order the elements of $R$ and $S$ into the two series, $\left\{x_{\xi}\right\}, \xi<\theta$, and $\left\{s_{\xi}\right\}, \xi<\theta$, where each element in $S$ appears $2^{N_{0}}$ times in the latter sequence, and $s_{\xi}$ is either of the form $\left(D_{\xi}, f_{\xi}, g_{\xi}\right)$ or $\left(D_{\xi}, f_{\xi}\right)$. Let $E^{*}$ be the set of $c$-condensation points of $E$ which are in $E, B^{*}=B \cap E^{*}$, and $D_{\xi}^{*}=D_{\xi} \cap E^{*}$. If $s_{\xi}=\left(D_{\xi}, f_{\xi}\right)$, define $J_{\xi}$ to be the set $J_{\xi}=\left\{x \mid f_{\xi}(x) \neq x, x \in D_{\xi}^{*}\right\}$. Since the power of the set $D_{\xi}-D_{\xi}^{*}$ is $<2 \aleph^{N_{0}}$, the power of $J_{\xi}$ is $2^{N_{0}}$.

If $s_{\gamma}$ is an element of $Q$, then as shown in Theorem 1.1 of [4], the power of the set $g_{\gamma}(E)-f_{\gamma}\left(D_{\gamma}\right)$ is $2^{N_{0}}$. From this it follows that the power of $g_{\gamma}\left(E^{*}\right)$ $-f_{\gamma}\left(D_{\gamma}\right)$ is $2^{N_{0}}$.

Suppose that $s_{0}$ is an element of $P$. Let $p_{0}$ be the first element in $J_{0}, t_{0}$ $=f_{0}\left(p_{0}\right)$, and let $q_{0}$ and $r_{0}$ represent no elements. Suppose that $s_{0}$ is an element of $Q$. Let $p_{0}$ and $q_{0}$ be the first two elements in the set $g_{0}\left(E^{*}\right)-f_{0}\left(D_{0}\right)$, and $r_{0}$ the first element in the set $f_{0}\left(D_{0}^{*}\right)-\left\{p_{0}, q_{0}\right\}$. Let $t_{0}$ represent no element. Now suppose that for each ordinal number $\xi<\mu$, where $\mu<\theta, p_{\xi}, q_{\xi}, r_{\xi}$, and $t_{\xi}$ have been defined. If $s_{\mu}$ is an element of $P$ let $p_{\mu}$ denote the first element in the set $J_{\mu}-\left[C_{\mu} \cup f_{\mu}^{*}\left(C_{\mu}\right)\right]$, where $P_{\mu}=\left\{p_{\xi} \mid \xi<\mu\right\}$ and

$$
C_{\mu}=\left\{q_{\xi}, r_{\xi}, t_{\xi} \mid \xi<\mu\right\} \cup P_{\mu} \cup \underset{\xi<\mu}{\cup} f_{\xi}\left(P_{\mu}\right) \cup \bigcup_{\xi<\mu} f_{\xi}^{*}\left(P_{\mu}\right) .
$$

Let $t_{\mu}=f_{\mu}\left(p_{\mu}\right)$ and $q_{\mu}$ and $r_{\mu}$ represent no elements. If $s_{\mu}$ is an element of $Q$ let $p_{\mu}$ and $q_{\mu}$ denote the first two elements in the set

$$
g_{\mu}\left(E^{*}\right)-\left[f_{\mu}\left(D_{\mu}\right) \cup\left\{p_{\xi}, q_{\xi}, r_{\xi}, t_{\xi} \mid \xi<\mu\right\} \cup \underset{\xi<\mu}{\cup} f_{\xi}\left(P_{\mu}\right) \cup \bigcup_{\xi<\mu} f_{\xi}^{*}\left(P_{\mu}\right)\right] .
$$

Denote by $r_{\mu}$ the first element in the set

$$
f_{\mu}\left(D_{\mu}^{*}\right)-\left[\left\{p_{\xi}, q_{\xi}, r_{\xi}, t_{\xi} \mid \xi<\mu\right\} \cup\left\{p_{\mu}, q_{\mu}\right\}\right] .
$$

Let $t_{\mu}$ represent no element.

Denote by $M$ the set $\left\{p_{\xi} \mid \xi<\theta\right\} \cap E^{*}$. Suppose that $F$ and $G$ are two dis- 
joint, similar subsets of $M$ of power $2^{\aleph_{0}}$ each. Let $f$ be a similarity transformation of $F$ onto $G$. By Lemma 1.1 of [4], $f$ can be extended to be an element $g$ of $L(D)$, for some element $D$, in $V(E)$, which contains $F$. For some ordinal number $\xi,(B, g)=\left(B_{\xi}, f_{\xi}\right)$. The argument given in Theorem 5 of [3] can be carried over to show that the set $M$ has property A. The argument given in Theorem 1.1 of [4] can be carried over to show that $\bar{M}$ and $\bar{B}$ are incomparable order types, and that $\bar{B}<\overline{M \cup B}<\bar{E}$. In fact, the arguments can be carried over to show that $\bar{M}$ and $\bar{B}^{*}$ are incomparable order types and that $\bar{B}^{*}<\overline{M \cup B}^{*}<\bar{E}^{*}$.

Now $M$ is a dense subset of $E^{*}$. This is so by the following reasoning. For each element $p$ in $E^{*}$ and each open interval $I$ of $R$, containing $p$, there exists a similarity transformation of $E$ into $R$ such that $f(x)=x$ for $x$ in $E-I$ and $f(x) \neq x$ for $x$ in $I \cap E$. Since $p$ is a $c$-condensation point of $E$, the function $f$ is in $L(E)$. Hence there are $2^{\aleph_{0}}$ elements in $E \cap I$. It follows that $M$ is a dense subset of $E$ which has property C. By Theorem 2.3 of [4], the set $M$ is exact.

If $E=R$, then the above construction of $M$ is modified slightly. The only variation from the above occurs if $J_{\mu}$ contains an open interval $I_{\mu}$ of $R$. In this case denote by $p_{\mu}$ the first element in the set

$$
N_{\mu}=I_{\mu}-\left[C_{\mu} \cup f_{\mu}^{*}\left(C_{\mu}\right) \cup f_{\mu}^{*}(B)\right] .
$$

Since $\bar{I}_{\mu} \equiv \lambda$ and $\overline{f_{\mu}{ }^{*}(B)}<\lambda$, the power of the set $N_{\mu}$ is $2^{\aleph_{0}}$. Consequently the element $p_{\mu}$ certainly exists. Suppose that $g$ is a similarity transformation of $N=M \cup B$ into $N$, which is not the identity transformation. As $M$ is a dense subset of $R, N$ is a dense subset of $R$. Thus the function $g$ can be extended to become a similarity transformation $h$ of $R$ into $R$. Since $h$ is not the identity transformation, $h$ is in $L(R)$. Thus, for some ordinal number $\delta$, $D_{\delta}=R$ and $h=f_{\delta}$. Now $g\left(p_{\delta}\right)=f_{\delta}\left(p_{\delta}\right)=t_{\delta}$. The element $t_{\delta}$ is not in $B$ and not in $M$, i.e., not in $N$. Therefore the function $g$ does not map $N$ into $N$, so that the set $N$ is exact.

CoROLlaRy. If the power of the order type $\alpha$ is $2^{N_{0}}$, and $\alpha<\lambda$, then there exists an order type $\beta$ with the following properties:

(1) $\beta$ has property A;

(2) $\alpha$ and $\beta$ are incomparable order types; and

(3) $\alpha<\alpha+\beta<\lambda$.

Remarks. (1) if $\bar{X} \leqq \bar{D}^{*}$, then the power of the set $E^{*}-X$ is $2^{N_{0}}$. This follows from the power of the set $E-E^{*}$ being $<2^{N_{0}}$.

(2) If $E$ has property $A$ and $B$ is a subset of $E$ for which the power of $E-B$ is $2^{N_{0}}$, then $E$ and $B$ satisfy the first group of hypotheses in Theorem 4.5. To see this suppose the contrary. Let $X$ be a set such that $\bar{X} \leqq \bar{B}$ and the power of $E-X$ is $<2^{N_{0}}$. As $\bar{X} \leqq \bar{B}$, there exists a similarity transformation $f$ of $X$ into $B$. Define $F$ to be the set $E \cap X$. Since the power of $E$ is $2^{N_{0}}$ and the power of $E-F$ is $\left\langle 2 \aleph^{N_{0}}\right.$, the power of $F$ is $2^{N_{0}}$. Combining this with the 
fact that the power of $E-F$ is $\left\langle 2^{\aleph_{0}}\right.$, we see that the power of the set $G=(E-B) \cap F$ is $2{ }^{N_{0}}$. Then $G$ and $f(G)$ are two disjoint, similar subsets of $E$, of power $2^{\aleph_{0}}$. This contradicts $E$ having property A.

(3) If $E$ is a proper subset of $R$, then it may not be possible for the set $M$ to be chosen so that $B \cup M$ is exact. An example will illustrate this point. Let $C$ and $D$ be dense subsets of the open intervals $(0,1 / 2)$ and $(1,2)$ respectively, such that $C \cup D$ has properties $\mathrm{A}$ and C. Let $f_{0}$ be the identity transformation on $C$. For each positive integer $n$, let $f_{n}$ be a similarity transformation, of the form $a x+b$, of $C$ into the open interval $(n /(n+1),(n+1) /(n+2))$. Let $B=\cup_{n<\omega} f_{n}(C)$, and $E=B \cup D$. The two sets, $E$ and $B$, satisfy the first two groups of hypotheses of Theorem 4.5. Nevertheless, for no subset $M$ of $E$ is $B \cup M$ exact.

\section{BIBLIOGRAPHY}

1. S. Banach, Sur les transformations biunivoques, Fund. Math. vol. 19 (1932) pp. 10-16.

2. B. Dushnik and E. W. Miller, Concerning similarity transformations of linearly ordered sets, Bull. Amer. Math. Soc. vol. 46 (1940) pp. 322-326.

3. S. Ginsburg, Order types and similarity transformations, submitted to Fund. Math.

4. - Some remarks on order types and decompositions of sets, Trans. Amer. Math. Soc. vol. 74 (1953) pp. 514-535.

5. E. Kamke, Theory of sets, New York, 1950.

6. G. Kurepa, Sur les ensembles ordonnés, Hrvatsko Prirodoslovno Društvo Glasnik Mat.Fiz. Astr. Ser II vol. 3 (1948) p. 146.

7. S. Mazurkiewicz, Sur la décomposition d'un. segment en une infinité d'ensembles nonmesurables superposables deux d deux, Fund. Math. vol. 2 (1921) pp. 8-14. $56-58$.

8. S. Ruziewicz, Sur une propriêté de la base hamelienne, Fund. Math. vol. 26 (1936) pp.

9. W. Sierpinski, Sur les translations des ensembles linéaires, Fund. Math. vol. 19 (1932) pp. 22-28. 550.

10. —, Un théorème de la théorie générale des ensembles, Fund. Math. vol. 25 (1935) p. 60. 164.

11. - Remarque sur les translations d'ensembles, Fund. Math. vol. 26 (1936) pp. 59-

12. - Sur les translations des ensembles linéaires, Fund. Math. vol. 35 (1948) pp. 159-

13. - Sur une décomposition de la droite, Comment. Math. Helv. vol. 22 (1949) pp. 317-320.

14. - Sur les types d'ordre des ensembles linéaires, Fund. Math. vol. 37 (1950) pp. 253-264.

15. J. von Neumann, Die Zerlegung eines Intervalles in abzählbar viele kongruente Teilmengen, Fund. Math. vol. 11 (1928) pp. 230-238.

UNIVERSITY OF MiAMI, Coral Gables, Fla. 